\title{
Luminescent solar concentrators. 1: Theory of operation and techniques for performance evaluation
}

\author{
J. S. Batchelder, A. H. Zewail, and T. Cole
}

\begin{abstract}
Techniques and calculations are presented that give explicit expressions for the over-all performance of a luminescent solar concentrator (LSC) in terms of the intrinsic spectral response and quantum efficiency of its constituents. We examine the single dye (or inorganic ion) LSC with emphasis on the planar geometry. Preliminary data on the degradation of candidate LSC dyes under severe weathering conditions are also given. Armed with our experimental results and analysis of solar absorption, self-absorption, and solar cell efficiency, we present a new genre of solar concentrator with a theory of operation for the device.
\end{abstract}

\section{Introduction}

A new concept in solar energy concentration technology has been evolving over the past several years, which has given rise to a device called a luminescent solar concentrator, or LSC (see Appendix A for an historical account). In our earlier paper ${ }^{1}$ we referred to the generic device as a planar solar concentrator, or PSC. However, since the optimal geometry for an LSC may well involve a nonplanar device, we shall use the general name of LSC for this entire classification of solar concentrator. The operation of an LSC is based on the idea of light pipe trapping of molecular or ionic luminescence. This trapped light can be coupled out of the LSC into photovoltaic cells (PVC) in such a way that the LSC provides a concentrated flux that is spectrally matched to the PVC so as to reduce the radiation heating and increase the electrical output of the PVC. An LSC does not need to track the sun and in fact can produce highly concentrated light output under either diffuse or direct insolation.

The conceptual operation of an LSC is illustrated by the diagram of a planar solar concentrator (PSC) shown in Fig. 1; a transparent material (e.g., polymethyl methacrylate, PMMA) is impregnated with guest luminescent absorbers (e.g., organic dye molecules) having strong absorption bands in the visible and UV regions

A. H. Zewail (to whom correspondence should be addressed) and J. S. Batchelder are with California Institute of Technology, Department of Applied Physics and A. A. Noyes Laboratory of Chemical Physics, Pasadena, California 91125. T. Cole is with Ford Motor Company, Engineering \& Research Staff, Dearborn, Michigan, 48121.

Received 13 April 1979.

0003-6935/79/183090-02\$00.50/0.

(C) 1979 Optical Society of America. of the spectrum, and also having an efficient quantum yield of emission. Solar photons entering the upper face of the plate are absorbed, and luminescent photons are then emitted. Snell's law dictates that a large fraction of these luminescent photons are trapped by total internal reflection; for example, about $74 \%$ of an isotropic emission will be trapped in a PMMA plate with an index of refraction of 1.49. Successive reflections transport the luminescent photons to the edge of the plate where they can enter an edge-mounted array of PVCs.

The photon flux at the edge of an idealized LSC is the product of the absorbed solar flux, the fraction of the resulting luminescence that is trapped, and the geometric ratio of the area of the face directly exposed to sunlight divided by the area of the edge that is covered by solar cells. Using the PSC of Fig. 1 as an example, a unit length of the plate which is $L$ units wide and $D$ units thick will have a geometric gain $G_{\text {geom }}$, which is given by

$$
G_{\text {geom }}=L / D=A_{f} / A_{e},
$$

where $A_{f}$ is the area of a face, and $A_{e}$ is the area of an edge. A typical PMMA plate is $3 \mathrm{~mm}$ thick, so that a square meter PSC section will have a geometric gain of $G_{\text {geom }}=333$. Such a gain exceeds the concentration of other known nontracking collectors using lenses or mirrors. Thus a high-cost high-efficiency solar cell can be coupled to this high-gain low-cost concentrator for a potentially low-cost system.

However, as nature usually dictates, a practical LSC will have a number of parasitic losses that limit the actual concentration to values lower than $G_{\text {geom }}$. Among these losses are inadequate absorption bandwidth, imperfect quantum efficiency, self-absorption of luminescence, absorption by the matrix material, reflective mismatches, geometrical trapping effects, and, of 


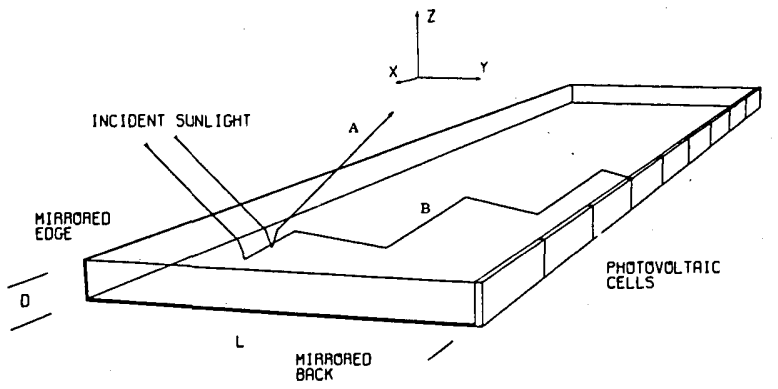

Fig. 1. A planar solar concentrator, or PSC, which is the particular embodiment of an LSC. ${ }^{10}$ Sunlight enters from above and passes twice through the plate thickness $D$, during which a dye of inorganic ion absorbs a certain portion of the solar flux. The ensuing luminescence can either escape back out of the face (A) or be trapped by total internal reflection (B). This trapped light will then propagate to the photovoltaic cells (PVC) where it is absorbed and converted into electricity.

course, the lifetime of the materials used. Clearly system optimization means close attention to minimizing these various losses.

In a previous publication ${ }^{1}$ we have demonstrated a method for overcoming one of the above losses-namely inadequate absorption bandwidth. For example, by including several dyes with successively overlapping absorption and emission bands, solar photons can be absorbed over the integrated absorption spectrum of all the dyes, with a cascade being formed by excitations being transferred from one dye to the next. In such a multiple-dye system the over-all efficiency will also depend on the mechanisms by which energy is transferred from one molecule to the next.

In this paper we shall present a formalism for expressing the operating characteristics of an LSC-PVC system in terms of measured molecular spectral responses and PVC characteristics. Preliminary experiments on LSC dye stability and self-absorption are reported. We will emphasize the methods used in calculating the performance of an LSC containing a single luminescent species and will typically use the PSC of Fig. 1 for illustrative calculations, although the formalism is developed in such a way so as to be readily adaptable to LSCs with more complex geometries containing multiple luminescent dyes or inorganic ions. In a subsequent paper we will treat the formalism of multiple-dye LSCs and nonplanar geometries, together with our experiments on prototype systems.

\section{Single Dye LSC Formalism}

Few processes in an LSC are subtle; an accurate calculation of the LSC output requires that the various channels that the photon can take be identified and weighted appropriately. A general description of these channels is given in the following section, and a pictorial flow chart is given in Fig. 2. Subsequently we develop relationships for the coefficients weighting each channel. For clarity we will typically discuss an LSC using

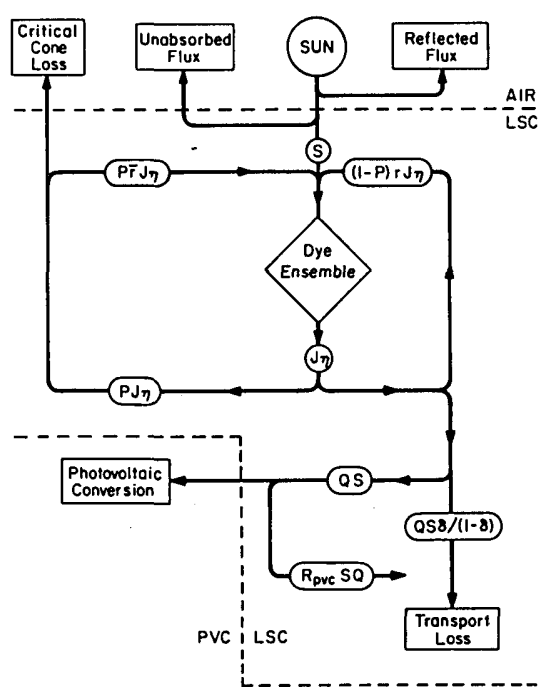

Fig. 2. A photon flow diagram depicting the predominant channels available in an LSC. Dotted lines represent changes in index of refraction, and squares represent photon sinks. Light from the sun enters the dye ensemble, resulting in luminescence that is converted in the PVC. The two feedback loops around the dye ensemble represent the effects of self-absorption inside and outside of the critical cones.

organic dyes in the PSC geometry of Fig. 1. However, unless explicitly stated the results are applicable to any absorber or geometry.

\section{A. Photon Flow Diagram for a Single Dye System}

We can trace the flow of excitations in an LSC with the aid of the flow chart in Fig. 1. Above all is the sun. Part of its incident flux will be lost directly by reflection from the LSC surface, and part is lost because its wavelength does not correspond to the absorption band of the dye used. What is left is the absorbed solar flux in the dye ensemble, denoted by $S$. There is a net rate of excitation $J$ of the dye ensemble, which in steady state must correspond to the rate of deexcitation. The photon output of the dye ensemble is the quantum efficiency of luminescence $\eta$ times $J$. This luminescence is geometrically divided into the fraction $J P \eta$, which is emitted within any of the critical escape cones, and the fraction $J(1-P) \eta$, which is trapped. For light within the critical cones there is an average probability $\bar{r}$ that self-absorption will take place before the light can escape out of the LSC, so that there is a feedback loop of magnitude $P \bar{r} J \eta$ of emissions in the critical cones that are recovered as excitations in the dye ensemble.

A similar feedback loop occurs with the self-absorption of light, which is trapped by total internal reflection; in this case the probability that a trapped photon will be self-absorbed before it reaches the LSC-PVC interface is $r$. An additional lumped parameter $\delta$ describes the fraction of the trapped luminescence which is lost due to matrix absorption or imperfect reflections. 
A hardy fraction $Q$ of the originally absorbed solar photons arrives at the LSC-PVC interface where a reflection takes place of magnitude $R_{p v c}$. In this analysis we will assume that such reflected flux is lost; to the extent that this assumption is not true we will underestimate the final output.

\section{B. Solar Absorption- $S$}

The solar spectrum is a variable quantity. The spectrum that we have used in our calculations is the Air Mass 1 (AM1) total incident radiation, measured on a clear cool summer day in Delaware. ${ }^{2}$ The spectral flux per wavenumber will be noted by $N(\bar{\nu})$ and the total flux by $I$, where

$$
I=\int_{0}^{\infty} N(\bar{\nu}) d \bar{\nu} .
$$

Since both the spectral distribution and the total flux vary considerably with atmospheric conditions, an accurate performance prediction requires that a solar spectrum be used that duplicates as closely as possible the sunlight that will be found under typical operating conditions. ${ }^{3}$

We will also use the normalized function $U\left(\theta_{i}, \bar{\nu}\right)$ for the angular distribution of the incident light, so that any combination of diffuse and direct sunlight can be modeled. In this case the total incident flux becomes

$$
I=\int_{0}^{\infty} d \bar{\nu} \int_{0}^{\pi / 2} d \theta_{i} N(\bar{\nu}) U\left(\theta_{i}, \bar{\nu}\right) \sin \left(\theta_{i}\right) \cos \left(\theta_{i}\right)
$$

with the normalization condition for $U\left(\theta_{i}, \bar{\nu}\right)$ given by

$$
1=\int_{0}^{\infty} d \bar{\nu} \int_{0}^{\pi / 2} d \theta_{i} U\left(\theta_{i}, \bar{\nu}\right) \sin \left(\theta_{i}\right) \cos \left(\theta_{i}\right) .
$$

A fraction of the sunlight will inevitably be reflected by the LSC surface. If no antireflection coating is used, the reflection coefficient $R\left(\theta_{i}\right)$ for unpolarized light is given by the Fresnel equation ${ }^{4}$

$$
\begin{aligned}
R\left(\theta_{i}\right) & =\frac{1}{2}\left[\frac{\tan ^{2}\left(\theta_{i}-\theta_{t}\right)}{\tan ^{2}\left(\theta_{i}+\theta_{t}\right)}+\frac{\sin ^{2}\left(\theta_{i}-\theta_{t}\right)}{\sin ^{2}\left(\theta_{i}+\theta_{t}\right)}\right], \\
\sin \left(\theta_{i}\right) & =n \sin \left(\theta_{t}\right),
\end{aligned}
$$

where $n$ is the index of refraction of the matrix material. The transmission coefficient $T\left(\theta_{i}\right)$ is given by

$$
T\left(\theta_{i}\right)=1-R\left(\theta_{i}\right) .
$$

Increasing the index of refraction of the matrix material increases the loss of sunlight due to surface reflections, but it also increases the fraction of the luminescence that is trapped by total internal reflection. The critical angle $\theta_{c}$ for total internal reflection is

$$
\theta_{c}=\sin ^{-1}(1 / n) \text {. }
$$

In Sec. II. D we show that in a planar LSC, such as in Fig. 1, this leads to the geometrical fraction $P$ of the luminescence that is emitted at an angle $\theta \leqslant \theta_{c}$, i.e., not trapped by total internal reflection, which is given by

$$
P=1-\left(1-1 / n^{2}\right)^{1 / 2} \text {. }
$$

that $T\left(\theta_{i}\right)(1-P)$ be maximized with respect to the index of refraction $n$. If no antireflection coating is used, this occurs at $n=2.5$

The addition of an antireflection coating might be desirable to remove some of the reflective loss, especially of higher index matrix materials are used. In this case the reflection coefficient for unpolarized light is $R\left(\theta_{i}, \vec{\nu}\right),{ }^{6}$ where

$$
\begin{aligned}
R\left(\theta_{i}, \bar{\nu}\right)= & \frac{1}{2}\left[\frac{r_{12}^{2}+r_{23}^{2}+2 r_{12} r_{23} \cos (2 \beta)}{1+r_{12}^{2} r_{23}^{2}+2 r_{12} r_{23} \cos (2 \beta)}\right. \\
& \left.+\frac{R_{12}^{2}+R_{23}^{2}+2 R_{12} R_{23} \cos (2 \beta)}{1+R_{12}^{2} R_{23}^{2}+2 R_{12} R_{23} \cos (2 \beta)}\right], \\
r_{12}= & \frac{\cos \left(\theta_{i}\right)-n_{1} \cos \left(\theta_{1}\right)}{\cos \left(\theta_{i}\right)+n_{1} \cos \left(\theta_{1}\right)}, \\
r_{23}= & \frac{n_{1} \cos \left(\theta_{1}\right)-n \cos \left(\theta_{t}\right)}{n_{1} \cos \left(\theta_{1}\right)+n \cos \left(\theta_{t}\right)}, \\
R_{12}= & \frac{n_{1} \cos \left(\theta_{1}\right)-\cos \left(\theta_{i}\right)}{n_{1} \cos \left(\theta_{i}\right)+\cos \left(\theta_{1}\right)}, \\
R_{23}= & \frac{n \cos \left(\theta_{t}\right)-n_{1} \cos \left(\theta_{1}\right)}{n \cos \left(\theta_{1}\right)+n_{1} \cos \left(\theta_{t}\right)}, \\
\beta= & 2 \pi n_{1} h \bar{\nu} \cos \left(\theta_{1}\right), \\
\sin \left(\theta_{i}\right)= & n_{1} \sin \left(\theta_{1}\right)=n \sin \left(\theta_{t}\right) .
\end{aligned}
$$

$n_{1}$ is the index of refraction of the antireflection coating, and $h$ is its thickness. Since the critical cone angle depends only on the index of the matrix material, adding an AR coating does not change the fraction of luminescence that is trapped, so that in this case the matrix index should be made as high as possible.

Numerical Example: Typical matrix materials such as PMMA have an index of refraction of about 1.5. The critical angle is found by Eq. (6) to be $\theta_{c}=\sin ^{-1}(1 / 1.5)$ $=42^{\circ}$, and the fraction of the luminescence that can escape out of the critical cones is $P=1-\left[1-\left(1 / 1.5^{2}\right]^{1 / 2}\right.$ $=0.255$ for a planar device. Without an antireflection coating, unpolarized light will be $96 \%$ transmitted at vertical incidence, and $94.2 \%$ is transmitted at an angle of incidence of $50^{\circ}$. If a $\mathrm{MgF}_{2}$ antireflection coating is used, with an index of 1.38 , the normal transmission is $98.5 \%$, and at $50^{\circ}$ the transmission is $97.4 \%$ for $6000-\AA$ light and a $1200-\AA$ coating thickness.

Passing the air-LSC interface, the light will be partially absorbed by both the dye molecules and the matrix material. We define an absorption coefficient for the dye $\alpha(\bar{\nu})$ :

$$
\alpha(\bar{\nu})=C \epsilon(\bar{\nu}) \ln (10),
$$

where $C$ is the molar dye concentration in the matrix material, and $\epsilon(\bar{\nu})$ is the molar extinction coefficient of the dye. The ratio of the transmitted intensity $I_{t}(\bar{\nu})$ to the initial intensity $I_{i}(\bar{\nu})$ over a path length $x$ is then

$$
\begin{aligned}
I_{t}(\bar{\nu}) / I_{i}(\bar{\nu}) & =\exp [-\alpha(\bar{\nu}) x] \\
& =10^{-} C_{\epsilon}(\bar{\nu}) x .
\end{aligned}
$$

Similarly let $\alpha_{m}(\bar{\nu})$ be the matrix absorption coefficient, so that the total combined absorption coefficient $\alpha_{t}(\bar{\nu})$ is

$$
\alpha_{t}(\bar{\nu})=\alpha(\bar{\nu})+\alpha_{m}(\bar{\nu}) .
$$


We now have sufficient information to determine the amount of solar flux $S$ that is absorbed by the dye per unit area of the LSC. In its most general form, $S$ is defined as follows:

$$
\begin{aligned}
S & =\int_{0}^{\infty} d \bar{\nu} \int_{0}^{\pi / 2} d \theta_{i} T\left(\theta_{i}, \bar{\nu}\right) N(\bar{\nu}) U\left(\theta_{i}, \bar{\nu}\right) \frac{\alpha(\bar{\nu})}{\alpha_{t}(\bar{\nu})} \\
& \times\left\{1-\exp \left[-\alpha_{t}(\bar{\nu}) l_{s}\right]\right\} \sin \left(\theta_{i}\right) \cos \left(\theta_{i}\right)
\end{aligned}
$$

This is just the incident solar flux of Eq. (3), $N(\bar{\nu})$, times the transmission coefficient for entering the LSC, $T\left(\theta_{i}, \bar{\nu}\right)$, times the fraction $\alpha(\bar{\nu}) / \alpha_{t}(\bar{\nu})$, which specifies how much of the absorbed flux was absorbed by the dye, times the total absorption probability $\left\{1-\exp \left[-\alpha_{t}(\bar{\nu})\right.\right.$ $\left.l_{s}\right]$, integrated over all angles of incidence and wavenumbers. $l_{s}$ is the path length traveled by the solar flux inside the LSC, which for the PSC geometry of Fig. 1 is given by

$$
l_{s}=2 D / \cos \left(\theta_{t}\right)=2 D /\left[1-\sin ^{2}\left(\theta_{i}\right) / n^{2}\right]^{1 / 2},
$$

where $D$ is the thickness of the plate. The factor of two appears in Eq. (12) due to a backing mirror, which causes the solar flux to pass twice through the plate. We have ignored the effect of multiple internal reflections of the sunlight by the LSC faces, which is shown in Appendix B to be a good approximation for moderate angles of incidence.

It is worthwhile now to ask what is the angular dependence of the solar absorption $S$ with respect to the incident light. From Eq. (11) it follows that $S$ is nearly proportional to the cosine of the angle of incidence, which means that the LSC behaves as a selectively absorbing blackbody. This tendency to imitate a blackbody is shown in the following example.

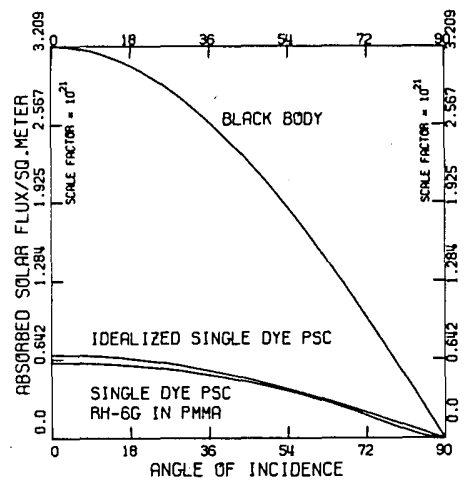

Fig. 3. The lowest curve shows the result of a numerical integration of Eq. (11) for an AM1 solar spectrum incident on a 3-mm thick LSC containing the laser dye rhodamine- $6 \mathrm{G}$ at a concentration of 0.001 moles/liter. The total absorbed flux is plotted as a function of the angle of incidence of the sunlight and is found to be remarkably similar to just the decrease in subtended area described by the cosine function. We can say that to a good approximation an idealized LSC will have the cosine dependence of a blackbody absorber but with a reduced total absorption (middle curve).
Numerical Example: The bottom curve in Fig. 3 represents the numerical integration of Eq. (11) for a 3-mm LSC plate containing a 0.001-M concentration of rhodamine-6G (Ref. 7) under AM1 insolation. The angle of incidence of the sunlight is varied using the angular distribution function $U\left(\theta_{i}, \bar{\nu}\right)$, treating the sun as a point source at a variable polar angle. The decrease in $S$ at large angles of incidence is due mainly to the decrease in the effective area exposed to the sun, given by the actual area times $\cos \left(\theta_{i}\right)$. For comparison, the center curve is a simple cosine function that is tangent to the calculated $S$ curve at about the Brewster angle, and the top curve shows the absorption by a perfect blackbody. This particular dye concentration and plate thickness combination absorb about $20 \%$ of the solar flux, which is the typical limit of a single dye device.

The fraction of the solar flux that is absorbed is quite sensitive to the concentration of the particular dye used. In the following example we calculate how $S$ varies with concentration in a typical system.

Numerical Example: Figure 4 shows the result of a numerical integration of Eq. (11) for a single pass 2-mm thick PMMA plate containing a variable concentration of rhodamine-6G for an AM1 spectrum at vertical incidence. This function can be approximated by a simple analytic expression containing two adjustable parameters which characterize the dye used, $K_{1}$ and $K_{2}$. This analytic approximation is given by

$$
S \cong K_{1} \cdot I\left[1-\exp \left(-K_{2} C l_{s}\right)\right] \text {, }
$$

where $C$ is the molar dye concentration, and $l_{s}$ is the path length traversed by the sunlight in the LSC. I is the total integrated solar flux. $K_{1}=0.20$, and $K_{2}=$ 6440. liters mole ${ }^{-1} \mathrm{~cm}^{-1}$ for a rhodamine-6G LSC.

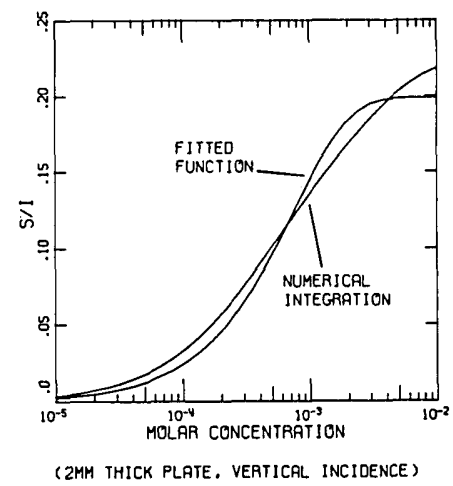

Fig. 4. The numerically integrated curve shows the result of solving Eq. (11) for the case of vertically incident sunlight on a single pass 2-mm PMMA plate containing a variable concentration of rhodamine-6G. A reasonable approximation to this result can be made using the form $S / I=K_{1}\left[1-\exp \left(-K_{2} C l_{s}\right)\right]$, where $C$ is the dye concentration in moles/liter, and $l_{s}$ is the path length of the sunlight in the LSC in centimeters. $K_{1}$ and $K_{2}$ are fitted constants, which for a rhodamine- $6 \mathrm{G}$ single dye LSC are given by $K_{1}=0.20$ and $K_{2}=$ 6440.0 liter mole $\mathrm{cm}^{-1}$. 


\section{Dye Quantum Efficiency- $\eta$}

Upon the absorption of a photon, a dye molecule will vibrationally relax to an excited singlet state on the time scale of picoseconds, or more slowly to the excited triplet state via intersystem crossing. Four major channels are then available by which the molecule can relax to its ground state: fluorescence from the excited singlet state; phosphorescence from the excited triplet state; direct or nonradiative transfer of the excitation to a nearby molecule; and finally by internal conversion of the excitation to molecular vibrations or phonons, which are dispersed in the lattice. In a single dye LSC the only direct transfer that can take place is to a similar dye molecule or to the matrix material, both of which effects are relatively negligible due to the dominance of intramolecular effects. So in a single dye LSC the important types of energy transfer to consider are fluorescence and phosphorescence (which are combined in the term luminescence) and internal conversion.

In this paper we define the quantum efficiency of luminescence as follows: if an isolated dye molecule in the matrix material of an LSC absorbs a photon of sufficient energy to excite the electronic state of the molecule to or above the first excited singlet state (but of insufficient energy to cause photodissociation), $\eta$ is the probability that the molecule will subsequently emit a photon. For dyes typically used in an LSC, $\eta$ will typically range from 0.85 to 0.95 .

When an excited dye molecule luminesces, there is a probability distribution $f(\bar{\nu})$ describing the wave-
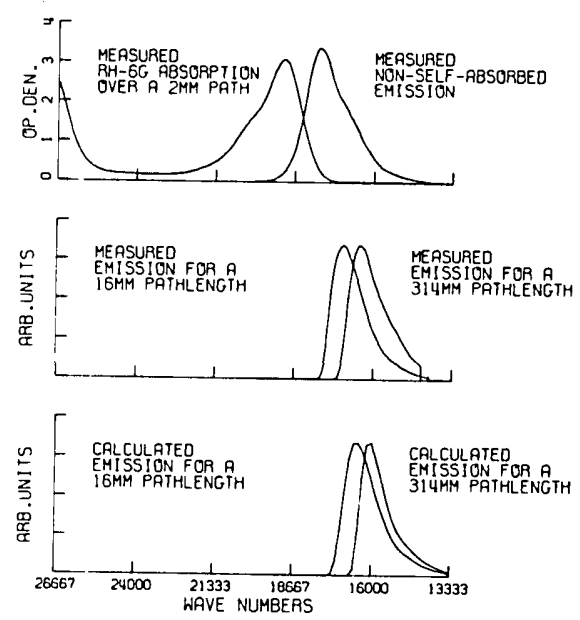

Fig. 5. The effect of self-absorption in a semi-infinite rod. A dye with the absorption and emission spectra shown in the upper graph is placed in a PMMA rod $2 \mathrm{~mm}$ in diameter and $400 \mathrm{~mm}$ long. Luminescent photons from molecules in the middle of the rod will undergo self-absorption on their way to the end of the rod. The cylindrical surface of the rod is roughened and blackened to eliminate internal reflections. The center graph shows the measured self-absorbed emission from the end of the rod for excitations originating $16 \mathrm{~mm}$ and $314 \mathrm{~mm}$ away from the end of the rod. The lower graph shows these same spectra as predicted by the self-absorption calculation of Sec. II.E. number of the emitted photon. $f(\bar{\nu})$ is called the normalized luminescence spectrum of the dye, and the normalization condition is given by

$$
\int_{0}^{\infty} f(\bar{\nu}) d \bar{\nu}=1
$$

\section{Losses via the Critical Cone-P}

Luminescence incident to the LSC surface at an angle of incidence greater than $\theta_{c}$ is totally internally reflected. The cone formed by all rays originating at the point of luminescence and forming an angle $\theta_{c}$ with the surface is called the critical cone. Typically in an LSC there will be two such cones, one pointing toward the top face and one towards the bottom. For an LSC which is not planar, the polar angle formed by these rays will be a function of depth $z$ and azimuthal angle $\phi$, so that, neglecting reflections at the air-LSC interface within the critical cones, the fraction of the luminescence lost out of the critical cone $P$ is

$$
P(z)=1-(4 \pi)^{-1} \int_{0}^{2 \pi} d \phi \int_{\theta_{c}(z, \phi)}^{\pi-\theta_{o}^{\prime}(z, \phi)} d \theta \sin \theta .
$$

In words this says that the fraction of the $4 \pi$ sphere of emission that escapes is one minus the probability that the angle of incidence will be greater than the critical angle. If either the top or bottom surface of the LSC is nonplanar, the polar angle of emission which will form a critical angle of incidence is not necessarily the critical angle, so that both the upper and lower critical cones require a variable limit on the $\theta$ integration. These limits are $\theta_{c}(\mathrm{z}, \phi)$ and $\theta_{c}^{\prime}(\mathrm{z}, \phi)$ for the upper and lower critical cones, respectively.

In the case of a planar geometry, the critical cones are independent of the depth and azimuthal angle, $\theta_{c}(z, \phi)$ $=\theta_{c}^{\prime}(z, \phi)=\theta_{c}$, and so the above integral becomes

$$
P=1-\cos \theta_{c} .
$$

If the index of refraction of air is assumed to be 1 , then $\theta_{c}=\sin ^{-1}(1 / n)$ from Eq. (6), and $P$ becomes

$$
P=1-\left(1-1 / n^{2}\right)^{1 / 2} \text {. }
$$

For example, $P=0.26$ for PMMA, which has an index of refraction of 1.49 .

Even within the critical cones, part of the luminescence can be retained via incomplete transmission at the air-LSC interface. As computed in Appendix B, this effect typically reduces $P$ by no more than $0.01 \%$.

\section{E. Self-Absorption Effects- $r, \bar{r}$}

A phenomenon with which we now have to concern ourselves is the fact that there is some overlap between the absorption and emission spectra for most of the dyes or ions to be used in an LSC. The upper graph of Fig. 5 shows the absorption spectrum of rhodamine-6G on the left, superimposed on its emission spectrum on the right. The observed overlap allows a fluorescent photon to be reabsorbed by another dye molecule of the same type. This effect has been seen in liquids in the form of secondary emission. ${ }^{8}$ Such a reabsorption is termed self-absorption and is actually a dominant effect over the long path lengths traveled by light trapped in 
an LSC (see Appendix D for the standard ${ }^{9}$ self-absorption treatment).

Self-absorption is a sensitive test of the line inhomogeneity and of the relative orientation of the respective donors and acceptors. We will presently ignore such complications and assume that at room temperature the entire lineshape can be thought of as homogeneous due to the rapid exchange that occurs between the various homogeneous ensembles. Specifically we will assume here that the measured absorption and emission spectra represent possible transitions for every dye molecule in the LSC.

We will calculate the effects of this self-absorption process via a collection efficiency $Q$, which is a generalization of the collection efficiency used by Weber and Lambe. ${ }^{10} Q$ is defined as the fraction of the absorbed solar photons that succeed in being transported to the LSC-PVC interface. As a simple example we can find $Q$ for an LSC having no self-absorption or transport loss $(\delta=0$, see Sec. II.F). No self-absorption is insured by requiring the possible emission frequencies to be exclusively less than all the possible absorption frequencies. In this case we find that of the solar photons absorbed by the dye ensemble, the fraction $\eta$ will be luminesced, and of those the fraction $(1-P)$ will be trapped via total internal reflection and transported to the LSC-PVC interface, so that in this idealized case $Q$ is given simply by

$$
Q_{\text {idealized }}=(1-P) \eta
$$

If we now turn on the self-absorption effect, the situation becomes more complicated. If we allow the $a b$ sorption and emission bands to overlap, the dye ensemble is capable of absorbing its own luminescence, and the LSC plate acts as a filter that attenuates the luminescence resulting from the absorption of solar photons. The plate is a peculiar sort of filter, however, in that the light that is absorbed can then be reluminesced in an arbitrary direction, most of which will again be trapped within the plate. Thus we will generate a number of different generations of luminescence: the first generation is the luminescence resulting from the initial absorption of the solar photons; the second generation is the luminescence resulting from the selfabsorption of first generation photons; the third generation is the luminescence resulting from the selfabsorption of the second generation photons; and so forth. An absorbed photon can be emitted with an energy less than, equal to, or a few $\mathrm{k} t$ greater than its initial energy, so that each generation is progressively red-shifted with respect to the preceding generation. Self-absorption will take place at higher rates farther from the LSC-PVC interface due to the longer average path lengths, so that the spatial distribution of all but the first generation will be nonuniform.

We shall subsequently develop a technique that enables the spatial and frequency distribution of all generations to be calculated from the geometry of an LSC and the absorption and emission spectra of the dye used. This calculation finds $Q$, the fraction of the initially absorbed solar photons that are transported to the
LSC-PVC interface, for an arbitrary geometry and dye in the limit of no transport loss. Prior to embarking on this adventure it is helpful to look at $Q$ for a simplified case. Starting with the PSC geometry of Fig. 1, we want to find the collection efficiency $Q^{(1)}$ of just the first generation emission for an arbitrary dye in the limit of no matrix absorption or reflection losses. Clearly the total collection efficiency $Q$ will have components from the collection of higher generation emissions, but this first generation collection efficiency will demonstrate many of the general principles involved in the full calculation. Due to self-absorption by the dye molecules, the first generation photon flux will be attenuated by a factor $\exp \left[-\alpha(\bar{\nu}) l_{s}\right]$, where $\alpha(\bar{\nu})$ is the absorption coefficient of the dye from Eq. (9), and $l_{s}$ is the path length along the photon's trajectory. $Q^{(1)}$ is obtained by integrating the probability of arrival for all paths from a given volume element, over all volume elements across the width $L$ of the PSC, and over all wave numbers of the emission weighted by the normalized luminescence spectrum of the dye $f(\bar{\nu})$ :

$$
\begin{aligned}
Q_{\mathrm{PSC}}^{(1)}= & \eta(2 \pi L)^{-1} \int_{0}^{\infty} f(\bar{\nu}) d \bar{\nu} \int_{0}^{L} d y \int_{0}^{\pi} d \phi \int_{\theta_{\mathrm{c}}}^{\pi / 2} \sin (\theta) d \theta \\
& \{\exp [-\alpha(\bar{\nu})(L-y) / \sin (\theta) \sin (\phi)] \\
& +\exp [-\alpha(\bar{\nu})(L+y) / \sin (\theta) \sin (\phi)]\} .
\end{aligned}
$$

This expression is similar to that given by Weber and Lambe ${ }^{10}$ except that we have defined $Q^{(1)}$ to include the dye quantum efficiency and not to include the Fresnel reflection that takes place at the LSC-PVC interface. The integral over $y$ can be obtained analytically, giving

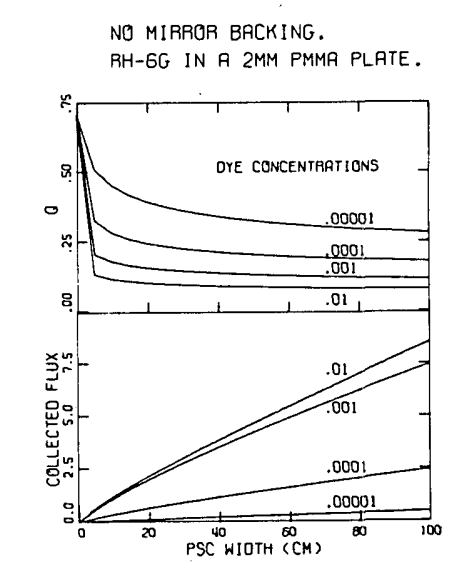

Fig. 6. The upper graph shows the approximate collection efficiency for a PSC in the limit that the reemission of self-absorbed luminescence is ignored. This collection efficiency $Q_{P S C}^{(1)}$ is calculated as a function of the width $L$ of the PSC for four different concentrations: $10^{-2}, 10^{-3}, 10^{-4}$, and $10^{-5}$ moles/liter of rhodamine-6G. The lower plot combines the information in the upper plot with that of Fig. 4 to find what the output flux is at the edge of the PSC in units of the total solar flux $I$ for the four different concentrations. The collected flux here is the number of photons per unit area that arrive at the edge in units of the total integrated solar flux per unit area. 


$$
\begin{aligned}
Q_{\mathrm{PSC}}^{(1)}= & \eta \pi^{-1} \int_{0}^{\infty} f(\bar{\nu}) d \bar{\nu} \int_{\theta_{c}}^{\pi / 2} \sin (\theta) d \theta \\
& \times \int_{0}^{\pi / 2} d \phi \sin (\theta) \sin (\phi) / \alpha(\bar{\nu}) L \\
& \{1-\exp [-2 \alpha(\bar{\nu}) L / \sin (\theta) \sin (\phi)]\} .
\end{aligned}
$$

In the following numerical example $Q_{P S C}^{(1)}$ is calculated for a particular dye as a function of the dye concentration and the width of the PSC.

Numerical Example: We have taken the rhodamine-6G absorption and emission spectra from Fig. 5 (top) and, assuming a peak extinction coefficient of $\epsilon_{\max }$ $=1.2 \times 10^{5}$ liter mole ${ }^{-1} \mathrm{~cm}^{-1}$, have numerically integrated Eq. (20) as a function of the PSC width for four different dye concentrations: $0.00001 \mathrm{M} ; 0.0001 \mathrm{M}$; $0.001 \mathrm{M}$; and $0.01 \mathrm{M}$. The results from these integrations are shown in the upper plot of Fig. 6. In the limit of a small PSC width, self-absorption becomes negligible due to the short path lengths, and so all the concentrations achieve the idealized $Q$ from Eq. (18) of $\eta$ $(1-P)$. As the width increases, the first generation collection efficiency dies away rapidly at first, approaching a steady state limit of about $8 \%$ for the very high path lengths or concentrations. In the lower plot is shown the product of the first generation collection efficiency of the upper plot times the solar flux $S$ absorbed per unit length taken from Fig. 4. The collected flux is plotted in units of the natural solar flux density. What we see is that, despite their lower collection efficiency, the high dye concentration single dye LSC performs better than the lower concentration devices due to the increased solar absorption. We have neglected higher generations, which will be included presently, and matrix and reflection losses, which are discussed in Sec. II. F. We have also ignored here the effects of concentration quenching.

We are going to find three related lumped parameters through an explicit self-absorption calculation. One parameter is the average probability $r$ that any emission from the dye in a single dye LSC outside of the critical cone will be self-absorbed before it reaches the PVC. Similarly $\bar{r}$ is the probability that fluorescence inside the critical cone will be self-absorbed before it escapes out of the LSC. These probabilities weight the selfabsorption feedback loops shown in Fig. 2. The third quantity we are interested in is $N_{e}$, the average number of radiative emissions undergone by a photon which arrives at the PVC. $N_{e}$ is used in the abbreviated performance calculation given in Sec. V.

We arrive at values for $r, \vec{r}$, and $N_{e}$ through a calculation of the collection efficiency $Q$ in the limit of no matrix absorption and reflective losses. $Q$ is composed of the sum (we assume here that coherence effects between the generations can be ignored) of the collection efficiency of the first generation emission $Q^{(1)}$, plus the fraction of $S$ which becomes a second generation emission times the collection efficiency of that generation, which are combined in the term $Q^{(2)}$, plus similar higher order terms:

$$
Q=Q^{(1)}+Q^{(2)}+Q^{(3)}+\ldots
$$

Thus $S Q^{(3)}$ is the total flux that reaches the LSC-PVC interface as a direct result of third generation emission. If the $Q$ 's associated with each generation are known, the average generation number $N_{e}$ is given by

$$
N_{e}=\sum_{i} i Q^{(i)} / Q
$$

Figure 2 shows that if we ignore transport losses in the form of matrix absorption, scattering centers, and incomplete internal reflection due to surface roughness, we see that the fraction of the first generation emission that reaches the LSC-PVC interface is $(1-r)(1-P)$, and that which is self-absorbed for the first time is $[\bar{r} P$ $+(1-P) r]$. The total number of photons in the first generation emission is $S \eta A_{f}$, and as a result of selfabsorption the total number in the second generation is $S[\bar{r} P+(1-P) r] \eta^{2} A_{f}$. This process repeats itself in a geometric series so that the total transmitted fraction $Q$ can be expressed in terms of $r, \bar{r}, \eta$, and $P$ :

$$
\begin{aligned}
Q= & (1-P)(1-r) \eta+(1-P)(1-r)[\bar{r} P+(1-P) r] \eta^{2} \\
& +(1-P)(1-r)[\bar{r} P+(1-P) r]^{2} \eta^{3}+\ldots, \\
Q= & \frac{(1-r)(1-P) \eta}{1-\eta[\bar{r} P+(1-P) r]} .
\end{aligned}
$$

$r$ and $\bar{r}$ are separated by calculating $Q^{\prime}$, the collected fraction of emission in a system where self-absorption in the critical cone has mathematically been turned off. In this case we set $\bar{r}=0$, and solving Eq. (23) for $r$ gives

$$
r=\frac{(1-P) \eta-Q^{\prime}}{\eta(1-P)\left(1-Q^{\prime}\right)} .
$$

With $r, Q$, and $Q^{\prime}$ known, it follows that $\bar{r}$ is given by

$$
\bar{r}=\frac{Q[1-\eta r(1-P)]-(1-r)(1-P) \eta}{Q P \eta} .
$$

$Q, Q^{\prime}, r, \bar{r}$, and $N_{e}$ are computed in this way in the absence of losses from matrix absorption and surface roughness. (These losses will be included in Sec. II. F.) We assume that $r, \bar{r}$, and $N_{e}$ are nearly constant over a range of device configurations, so that only a few cases need actually be computed.

We use the following technique of computing $Q$ for an arbitrary geometry LSC. Absorption of incident light will create a distribution of excitations in the LSC which is specified by $\Xi^{(1)}(\bar{x}, \bar{\nu})$, the number of first generation excitations per unit volume and wave number. Integrating the excitation distribution function over the volume of the LSC and over all wave numbers yields the total number of absorbed solar photons $S A_{f}$ :

$$
S A_{f}=\int_{0}^{\infty} d \bar{\nu} \int_{\mathrm{LSC}} d^{3} x \Xi^{(1)}(\bar{x}, \bar{\nu})
$$

$\bar{\nu}$ in the excitation distribution function is the wave number of the photon when it was absorbed. Such a photon can be emitted at energies lower than $h c \bar{\nu}$, but can only be emitted at energies a few $\mathrm{kT}$ higher than $h c \bar{\nu}$. We account for this shift by allowing excitations of an energy greater than or equal to $h c \bar{\nu}$ to emit into a 
spectrum which has zero intensity for energies greater than $h c \bar{\nu}$ and is normalized and proportional to $f(\bar{\nu})$ for energies equal to or less than $h c \bar{\nu}$. For example, the first generation emission distribution $f^{(1)}(\bar{x}, \bar{\nu})$ is in units of excitations per unit volume and wave number and is given by the following expression:

$$
f^{(1)}(\bar{x}, \bar{\nu})=\eta f(\bar{\nu}) \int_{\bar{\nu}}^{\infty} d \bar{\nu}^{\prime} \Xi \bar{\Xi}^{(1)}\left(\bar{x}, \bar{\nu}^{\prime}\right) / \int_{0}^{\bar{\nu}^{\prime}} d \bar{\nu}^{\prime \prime} f\left(\bar{\nu}^{\prime \prime}\right) .
$$

The integral in the denominator serves to normalize the truncated emission spectrum. This emission is then partially absorbed by the dye ensemble, creating a second excitation distribution $\Xi^{(2)}(\bar{x}, \bar{\nu})$. Let $\Lambda(\bar{x}, \bar{y}, \bar{\nu})$ denote the probability per unit volume that an emission at a position $\bar{y}$ and wavenumber $\bar{\nu}$ will be absorbed at a position $\bar{x}$. The second generation excitation distribution is then the product of this filter function $\Lambda(\bar{x}, \bar{y}, \bar{\nu})$ and the first generation emission distribution $f^{(1)}(\bar{x}, \bar{\nu})$ integrated over the LSC volume:

$$
\Xi^{(2)}(\bar{x}, \bar{\nu})=\int d^{3} y \Lambda(\bar{x}, \bar{y}, \bar{\nu}) f^{(1)}(\bar{y}, \bar{\nu}) .
$$

Inserting the definition of $f^{(1)}(\bar{x}, \bar{\nu})$ from Eq. (27) into Eq. (28), we get a recursion relation for the excitation distribution functions. Such a recursion relation connects every generation with its predecessor, so that the $n$th excitation distribution function is defined in terms of the preceding distribution as follows:

$$
\begin{aligned}
\Xi^{(n)}(\bar{x}, \bar{\nu})= & f(\bar{\nu}) \eta \int_{\bar{\nu}}^{\infty} d \bar{\nu}^{\prime}\left[\int_{0}^{\bar{\nu}^{\prime}} f\left(\bar{\nu}^{\prime \prime}\right) d \bar{\nu}^{\prime \prime}\right]^{-1} \\
& \int d^{3} y \Lambda(\bar{x}, \bar{y}, \bar{\nu}) \Xi^{(n-1)}\left(\bar{y}, \bar{\nu}^{\prime}\right)
\end{aligned}
$$

Now we need a bookkeeping apparatus to keep track of the part of each generation of emission that is absorbed by the PVC. $Q^{(n)}$ is the fraction of the original $S$ excitations that is collected at the PVC due to the nonself-absorbed emission from the $n$th generation. Consider a PVC area element $d A$ located at a position $\bar{y}$ in space. This area element has a normal unit vector associated with it pointing away from the LSC, $\bar{A}(\bar{y})$, such that the effective area of the PVC element at $\bar{y}$ seen by a point of emission at $\bar{x}$ is given by $d A$ times the dot product of $\bar{A}(\bar{y})$ and a unit vector in the direction $(\bar{x}-$ $\bar{y}):[\bar{A}(\bar{y}) \cdot(\bar{x}-\bar{y})] d A /|\bar{x}-\bar{y}|$. Emission traveling from $\bar{x}$ to $\bar{y}$ will be attenuated by the path length squared, $\mid \bar{x}$ $-\left.\bar{y}\right|^{-2}$, as well as by the Beer-Lambert law absorption $\exp [-\alpha(\bar{\nu})|\bar{x}-\bar{y}|] . \quad Q^{(n)}$ is then the fraction of the emitted $n$th generation emission, $f^{(n)}(\bar{x}, \bar{\nu})$, which is successfully transmitted to a PVC area element $d A$, summed over all real and image emission points, all PVC elements, and wave numbers:

$$
\begin{aligned}
Q^{(n)}= & \int_{0}^{\infty} d \bar{\nu} \int_{\mathrm{PVC}} d A(\bar{y}) \\
& \times \int_{\mathrm{LSC}} d^{3} x f^{(n)}(\bar{x}, \bar{\nu}) \exp [-\alpha(\bar{\nu})|\bar{x}-\bar{y}|] \\
& {[\bar{A}(\bar{y}) \cdot(\bar{x}-\bar{y})] /\left[S|\bar{x}-\bar{y}|^{3} A_{e} A_{f}\right] }
\end{aligned}
$$

Combining this result with Eq. (21) and Eq. (27), we find that the total fraction collected $Q$ is the following sum of integrals of the excitation distribution functions:

$$
\begin{aligned}
Q= & \eta \int_{0}^{\infty} d \bar{\nu} f(\bar{\nu}) \int_{\mathrm{PVC}} d A(\bar{y}) \int_{\mathrm{LSC}} d^{3} x \exp [-\alpha(\bar{\nu})|\bar{x}-\bar{y}|] \\
& \left\{[\bar{A}(\bar{y}) \cdot(\bar{x}-\bar{y})] /\left(S|\bar{x}-\bar{y}|^{3} A_{e} A_{f}\right)\right\} \\
& \cdot\left\{\int_{\bar{\nu}}^{\infty} d \bar{\nu}^{\prime}\left[\sum_{n=1}^{\infty} \Xi{ }^{(n)}\left(\bar{x}, \bar{\nu}^{\prime}\right) / \int_{0}^{\bar{\nu}^{\prime}} f\left(\bar{\nu}^{\prime \prime}\right) d \bar{\nu}^{\prime \prime}\right]\right\}
\end{aligned}
$$

Thus the collection efficiency $Q$ is completely determined by the initial excitation distribution, $\Xi^{(1)}(\bar{x}, \vec{\nu})$, and by the filter function, $\Lambda(\bar{x}, \bar{y}, \bar{\nu})$, or the probability per unit volume that an emission at a position $\bar{y}$ and wavenumber $\bar{\nu}$ will be absorbed at a position $\bar{x}$. The collection efficiency $Q^{\prime}$ in the absence of self-absorption in the critical cone is computed in the same way, except that a new filter function $\Lambda^{\prime}(\bar{x}, \bar{y}, \bar{\nu})$ is used that ignores the possibility that emission inside the critical cones can be self-absorbed.

Further development of a generalized self-absorption formalism is less than revealing, in that almost immediately the symmetries of a particular geometry can be used to simplify the series of integral equations contained in Eq. (31). Two particular cases are of special interest here: one is a semi-infinite rod with a completely lossy (blackened) surface; and the second is the PSC geometry. The first case is sufficiently simple as to allow straightforward calculations, while the PSC is typical of a high symmetry LSC for which a numerical calculation is tractable.

\section{Lossy Semi-Infinite Rod: An Experimental Test}

Figure 7 shows a semi-infinite rod of LSC material located on the positive $x$ axis, whose diameter is $d$, which is connected to a PVC absorbing disk located at the origin and whose surface is totally absorbing. The rod is divided into a series of disks, each $d x$ long. The filter function in this geometry is the probability per unit length that emission by an element at $y$ with a wavenumber $\bar{\nu}$ will be absorbed by a disk at $x$ :

$$
\begin{aligned}
\Lambda(x, y, \bar{\nu})= & \alpha(\bar{\nu}) d^{2} \exp (-\alpha(\bar{\nu}) \cdot|x-y|) \\
& / 8\left((x-y)^{2}+d^{2} / 4\right)
\end{aligned}
$$

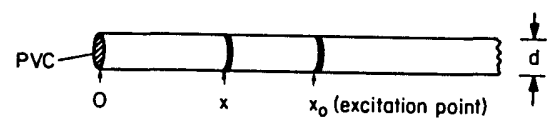

Fig. 7. The semi-infinite LSC rod geometry used in the sample self-absorption calculation given in the text. The rod is composed of a cylinder of diameter $d$ whose surface is completely absorbing and which terminates on a PVC disk at the origin. The initial excitation distribution is assumed to be contained in a disk located a distance $x_{0}$ down the rod. 
In other words, $\Lambda(x, y, \bar{\nu})$ is the probability per unit length that an emission from $y$ will be absorbed by a disk at $x$, with the approximation that no absorption occurs due to radial propagation. This integral form of the filter function is included for clarity; however, in a numerical calculation the finite length of the disk element as well as radial absorption must be taken into account.

Assume a point source monochromatic excitation at a position $x_{0}$ along the rod, at a wave number $\vec{\nu}_{0}$ greater than any possible emission wave number. If $S$ excitations are absorbed in a disk element of length $d x$, we find that the initial excitation distribution function, in excitations per unit length and wave number, is given by

$$
\Xi^{(1)}(x, \bar{\nu})=S \delta\left(x-x_{0}\right) \delta\left(\bar{\nu}-\bar{\nu}_{0}\right),
$$

where $\delta\left(x-x_{0}\right)$ and $\delta\left(\bar{\nu}-\bar{\nu}_{0}\right)$ are Dirac $\delta$ functions. For example, the first emission distribution is specified by Eq. (27) to be

$$
\begin{aligned}
f^{(1)}(x, \bar{\nu}) & =\eta f(\bar{\nu}) \int_{\bar{\nu}}^{\infty} d \bar{\nu}^{\prime} S \delta\left(x-x_{0}\right) \delta\left(\bar{\nu}^{\prime}-\bar{\nu}_{0}\right) / \int_{0}^{\bar{\nu}^{\prime}} d \bar{\nu}^{\prime \prime} f\left(\bar{\nu}^{\prime \prime}\right) \\
& =\eta f(\bar{\nu}) S \delta\left(x-x_{0}\right)
\end{aligned}
$$

The second generation excitation distribution is found by Eq. (28) to be

$$
\begin{aligned}
\Xi^{(2)}(x, \bar{\nu}) & =\int_{0}^{\infty} d y \Lambda(x, y, \bar{\nu}) f(\bar{\nu}) \delta\left(y-x_{0}\right) S \eta \\
& =\Lambda\left(x, x_{0}, \bar{\nu}\right) f(\bar{\nu}) S \eta .
\end{aligned}
$$

Thus for this geometry the excitation distribution recursion relation is

$$
\begin{aligned}
\Xi^{(n)}(x, \bar{\nu})= & f(\bar{\nu}) \eta \int_{0}^{\infty} d y \Lambda(x, y, \bar{\nu}) \\
& \cdot \int_{\bar{\nu}}^{\infty} \Xi^{(n-1)}\left(y, \bar{\nu}^{\prime}\right) d \bar{\nu}^{\prime} / \int_{0}^{\bar{\nu}^{\prime}} f\left(\bar{\nu}^{\prime \prime}\right) d \bar{\nu}^{\prime \prime}
\end{aligned}
$$

There is only one PVC element to consider for the collection efficiency $Q$, and its solid angle fraction of the total $4 \pi$ emission from $x$ is $a(x)=\left\{1-x /\left(d^{2} / 4+\right.\right.$ $\left.\left.x^{2}\right)^{1 / 2}\right\} / 2$, so that for this geometry Eq. (31) becomes

$$
\begin{aligned}
Q= & \eta \int_{0}^{\infty} d \bar{\nu} f(\bar{\nu}) \int_{0}^{\infty} d x \exp (-\alpha(\bar{\nu}) x) a(x) / S \\
& \cdot\left[\int_{\bar{\nu}}^{\infty} d \bar{\nu}^{\prime} \sum_{n=1}^{\infty} \Xi^{(n)}\left(x, \bar{\nu}^{\prime}\right) / \int_{0}^{\bar{\nu}^{\prime}} f\left(\bar{\nu}^{\prime \prime}\right) d \bar{\nu}^{\prime \prime}\right] .
\end{aligned}
$$

Finally we need $Q^{\prime}$, the collection efficiency for the case where self-absorption in the critical cones has been turned off. Since the surface of the rod is completely lossy, the critical cone extends over all emissions except those which intercept the PVC at the end of the rod. For $x_{0} \gg d$, the solid angle subtended by the PVC at typical points of emission is very small, so that we can ignore the contribution from emission resulting from self-absorption outside of the critical cone. What remains is the fraction of the first generation emission that reaches the PVC:

$$
Q^{\prime} \cong Q^{(1)}
$$

We have now solved for all the terms that are required to calculate $r, \bar{r}$, and $N_{e}$. The following example compares the results of this analysis with the actually measured spectra from the end of a blackened LSC rod illuminated at a point along the rod.

Numerical Example: The absorption and nonselfabsorbed emission spectra were measured for commercially produced PMMA containing the laser dye rhodamine-6G, as shown in the upper plot of Fig. 5. A $2-\mathrm{mm} \times 400-\mathrm{mm}$ rod of this material was everywhere roughened and blackened except for the ends, which were polished. An argon ion laser at $20,490 \mathrm{~cm}^{-1}(4880$ $\AA$ ) was used to create a point excitation at $16 \mathrm{~mm}$ or 314 $\mathrm{mm}$ away from the tip of the rod, and the luminescence spectra emerging from the tip were measured and is shown in the center plot of Fig. 5. The measured absorption and nonself-absorbed emission spectra were used to calculate the excitation distribution functions and filter function for this sample at $x_{0}=16 \mathrm{~mm}$ and $314 \mathrm{~mm}$. If these functions are inserted in Eq. (37) without performing the final integration over wave number, we obtain the spectrum of the output luminescence $Q(\bar{\nu})$. These computed spectra are shown in the lower plot of Fig. 5, in agreement with the measured spectra in the center plot. For an excitation at $16 \mathrm{~mm}$ we find $Q=5.7 \times 10^{-4}, Q^{\prime}=3.3 \times 10^{-4}, r=0.6, \bar{r}=0.4$, and $N_{e}=1.5$. With $x_{0}=314 \mathrm{~mm}$ we have that $Q=7.9$ $\times 10^{-7}, Q^{\prime}=4.8 \times 10^{-7}, r=0.8, \bar{r}=0.4$, and $N_{e}=1.5$ (all values only for a lossy rod).

\section{PSC Geometry}

We divide the PSC of Fig. 1 into a 1-D array of strips parallel to the PVC. This technique gives the simplest form for the filter and redistribution functions, although they can only be applied to systems whose initial excitation varies only across the width of the device. If the mirror is located at $x=0$, an emission at $x$ will cause an image emission at $-x$. The filter function $\Lambda(x, y, \bar{\nu})$ is found in a similar manner as Eq. (32) to be

$$
\begin{aligned}
\Lambda(x, y, \bar{\nu})= & \frac{\alpha(\bar{\nu})}{2 \pi} \int_{0}^{\pi} \frac{d \phi}{\sin (\phi)} \int_{0_{c}}^{\pi / 2} d \theta \\
& \cdot[\exp (-\alpha(\bar{\nu})|x-y| / \sin (\theta) \sin (\phi)) \\
& +\exp (-\alpha(\bar{\nu})|x+y| / \sin (\theta) \sin (\phi))] .
\end{aligned}
$$

The excitation distribution recursion relation is actually identical to that for the semi-infinite rod, Eq. (36), except that the integration is from 0 to the width $L$, rather than to infinity:

$$
\begin{aligned}
\Xi^{(n)}(x, \bar{\nu})= & \eta f(\bar{\nu}) \int_{0}^{L} d y \\
& \Lambda(x, y, \bar{\nu}) \int_{\bar{\nu}}^{\infty} \Xi^{(n-1)}\left(y, \bar{\nu}^{\prime}\right) d \bar{\nu}^{\prime} / \int_{0}^{\bar{\nu}^{\prime}} f\left(\bar{\nu}^{\prime \prime}\right) d \bar{\nu}^{\prime \prime} .
\end{aligned}
$$


The collection efficiency is an extension of the first generation collection efficiency found in Eq. (19), using the form of Eq. (31). Without further derivation, we find $Q$ for the PSC geometry is given by

$$
\begin{aligned}
Q= & \frac{\eta}{2 \pi}\left\{\int_{0}^{\infty} f(\bar{\nu}) d \bar{\nu} \int_{0}^{L} d x \int_{0}^{\pi} d \phi \int_{\theta_{c}}^{\pi 2} d \theta \sin (\theta)\right. \\
& \cdot[\exp (-\alpha(\bar{\nu})(L-x) / \sin (\theta) \sin (\phi)] \\
& +\exp (-\alpha(\bar{\nu})(L+x) / \sin (\theta) \sin (\phi))] \\
& \left.\cdot \int_{\bar{\nu}}^{\infty} d \bar{\nu}^{\prime} \sum_{n=1}^{\infty} \Xi^{(n)}\left(x, \bar{\nu}^{\prime}\right) / \int_{0}^{\bar{\nu}^{\prime}} f\left(\bar{\nu}^{\prime \prime}\right) d \bar{\nu}^{\prime \prime}\right\} \\
& / \int_{0}^{L} d x \int_{0}^{\infty} d \bar{\nu} \Xi^{(1)}(x, \bar{\nu}) .
\end{aligned}
$$

While these functions cannot be integrated analytically, they are readily tabulated numerically. Using a given dye's absorption and emission spectra as input, this technique predicts the effects of self-absorption for a given excitation and concentration. Results of such calculations. will be presented in a future publication.

\section{F. Photon Transport to the Absorber-Q.}

In Sec. II. $\mathrm{E}$ there are explicit calculations of the fraction $Q$ of the absorbed solar photons that arrive at the PVC absorber, ignoring effects of scattering centers, internal reflection losses, and other sample dependent loss mechanisms. In general an LSC will not be perfect, and so we introduce a term to take into account these loss mechanisms when computing the real $Q$ of a system.

If the above mentioned loss mechanisms are ignored, we have shown that $Q$ is given by the following [Eq. (23)]:

$$
Q=\frac{(1-r)(1-P) \eta}{1-\eta[\bar{r} P+(1-P) r]}
$$

We now introduce the loss term $\delta$ which gauges the collecting quality of a particular plate independent of the dyes used. $\delta$ is the fraction of the collectable luminescence which is lost due to such mechanisms as matrix absorption, scattering centers, and incomplete total internal reflection due to surface roughness and undulations. The final collected fraction of the absorbed solar flux is given by

$$
Q=\frac{(1-\delta)(1-r)(1-P) \eta}{1-\eta[\bar{r} P+(1-P) r]}
$$

Of the contributions that make up the transport loss $\delta$, the dominant one is absorption by the matrix material, followed by a variety of other mechanisms which tend to be sample dependent.

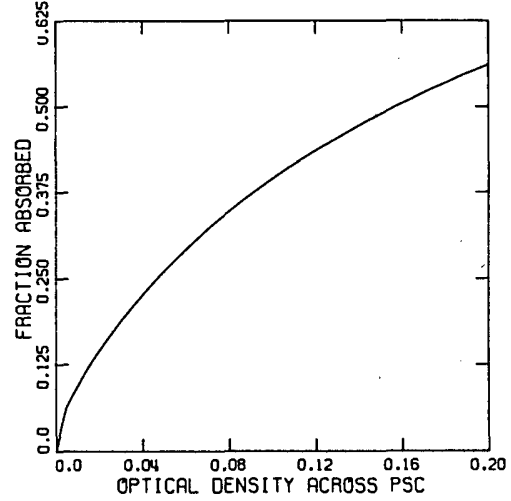

Fig. 8. The fraction of the trapped luminescence in an idealized PSC that is lost during transport to the PVC via matrix absorption. Assuming uniform initial illumination and ignoring the possibility of self-absorption and internal reflection losses from surface roughness, a finite element analysis is used to compute the emission that arrives at the edge of the PSC for a number of different optical densities of the matrix material. The details of the calculation are given in Appendix $\mathrm{C}$.

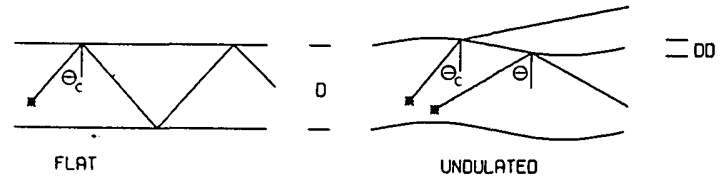

Fig. 9. The effect of surface undulations on the trapped light. If the surface of a planar LSC is not entirely flat, but has undulations over a characteristic distance $D D$, there will be convex portions of the surface where light which would have otherwise been trapped can escape. If the undulation is slow compared to the thickness of the plate, the altered critical cone loss $P_{\text {und }}$ is given by Eq. (43) for a plate of thickness $D$. Typical manufacturing tolerances for the flatness of these plates can be such that this effect should be negligible.

Numerical Example: The contribution to $\delta$ from matrix absorption in a typical LSC depends on the matrix material and the wavelength of the final emission. PMMA has an absorption coefficient of about 2 $\times 10^{-3} \mathrm{~cm}^{-1}$ in the neighborhood of $7000 \AA$, so that a PSC $50 \mathrm{~cm}$ across containing a dye whose final emission is centered at $7000 \AA$ will have a matrix optical density of about 0.04 across the width of the PSC. Figure 8 shows the calculated value of $\delta$ for different optical densities from a finite element analysis given in Appendix $\mathrm{C}$, so that a PSC with an optical density of 0.04 
will have a collection loss of about $20 \%$. Undulations in the surface will increase the effect size of the critical cones in the convex regions, allowing additional light to escape out of the surface. For undulations whose wavelengths are long compared to the thickness of the LSC, it can be shown that the effective critical cone loss in the undulating plate will be

$$
P_{\text {und. }}=1-\cos \left(\theta_{c}\right) /(1+D D / D) \text {, }
$$

where $D$ is the plate thickness, and $D D$ is the height of the undulation, as shown in Fig. 9. If the thickness of a 2-mm PMMA plate varies by $0.05 \mathrm{~mm}$ across the plate, the probability of escape out of the critical cones increases from 0.26 to 0.28 , giving a contribution to the transport loss of about $3 \%$.

Surface blemishes also generate paths for light escape. However, their characteristics and density will be peculiar to the materials used and the specific conditions of weathering. We will assume that the contribution from surface blemishes is small, considering the excellent weathering characteristics demonstrated by the materials being considered for use as LSCs. ${ }^{11}$

\section{G. Rate of Excitation of the Dye Ensemble-J, $W$}

Now that we have introduced a number of quantities- $S, P, \eta, r$, and so on-we need a technique to combine these various factors. One that we have found useful is to consider the rate of excitation of the dye ensemble, which we call $J$. Consider the dye ensemble as a collection of two state systems. $N$ is the total number of dye molecules, $n_{1}$ is the ground state population, and $n_{2}$ is the excited state population, such that

$$
N=n_{1}+n_{2} .
$$

The rate of photon production by the dye is the product of the number of molecules in the excited manifold times the spontaneous radiative transition probability as long as no stimulated emission takes place. Let $W_{12}$ be the total probability of deexcitation per second, and $W_{21}$ be the total probability of deexcitation per second. By detailed balance at steady state it must be true that

$$
n_{1} W_{12}=n_{2} W_{21} \text {. }
$$

Typically the system is unsaturated, or $n_{1} \gg n_{2}$. Then the rate of photon production by the dye is

$$
\eta W_{21} n_{2}=\eta W_{12}\left(N-n_{2}\right)=\eta W_{12} N .
$$

So we can define the rate of excitation $J$ as

$$
J=W_{12} N \text {. }
$$

From Fig. 2 it is clear that there are three sources of excitation: the initial solar input, the luminescence that is reabsorbed outside of the critical cones, and that which is reabsorbed inside the critical cones:

$$
\begin{aligned}
& J=S A_{f}+J \eta r(1-P)+J \eta \bar{r} P \\
& J=S A_{f} /\{1-\eta[\bar{r} P+(1-P) r]\} .
\end{aligned}
$$

The fraction of this excitation which arrives at the PVC will be the quantum efficiency of luminescence times that which is left after the losses from the critical cone, self-absorption, and transport. The total flux delivered to the PVC is called $W$ and from the above is given by

$$
\begin{aligned}
& W=J \eta(1-P)(1-r)(1-\delta), \\
& W=S A_{f} \eta(1-P)(1-r)(1-\delta) /\{1-\eta[\bar{r} P+(1-P) r]\} .
\end{aligned}
$$

Clearly we should arrive at the same result by observing that the collected flux $W$ is just the absorbed solar radiation $S A_{f}$ times the probability of collection $Q$ :

$$
W=S A_{f} Q .
$$

Combining this with the definition of $Q$ from Eq. (42) does in fact give Eq. (49).

\section{H. PVC Response- $V_{o c}$, F.F., $R_{\text {pvc }}$}

Light that finally reaches a photovoltaic cell (PVC) at the edge of an LSC will not necessarily be converted into electricity. The reflections that occur at the LSC-PVC interface are nonnegligible due to the high index of refraction of the PVC and to the large angle of emission typical of LSC output light. For example, the refractive indices of silicon and gallium arsenide near their bandgaps are about 3.5, so that an uncoated PVC exposed directly to the sun will reflect about $30 \%$ of the incident flux. It has been found that a good antireflection coating can substantially aid transmission of such direct light, making possible surface reflectivity of as low as 3\% (Ref. 6) between the semiconductor and air. Though the LSC would promise even better transmission into the PVC than is possible with an air-PVC interface due to the favorable index match between the LSC and the PVC, the output light from an LSC has a very high average angle of incidence, so that some of the benefit of the LSC's higher index is lost. If we calculate the intensity of light arriving at the PVC as a function of the angle of incidence to the PVC, we

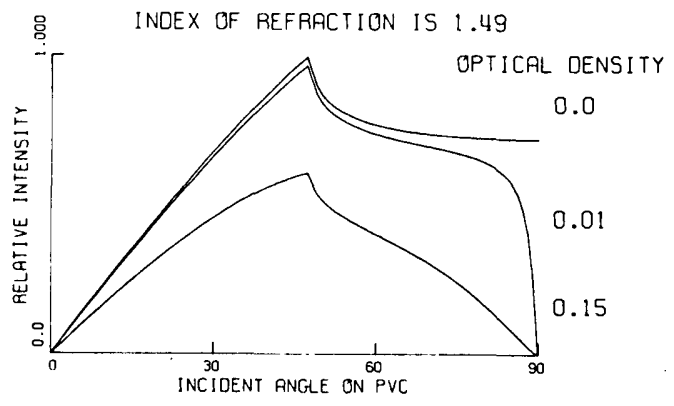

Fig. 10. The results of a finite element analysis developed in Appendix $\mathrm{C}$ for the intensity arriving at the edge of a PSC as a function of the angle of incidence to the plane of the edge. The intensity peaks at the compliment of the critical angle and extends to very large angles of incidence due to the infinite strip geometry of the PSC. The optical densities shown are for the matrix material measured across the width of the PSC. 
find that for a typical planar LSC the intensity is greatest at the compliment of the critical angle. The actual angular distribution changes with LSC geometry, and its form is necessary for an accurate calculation of the reflection losses incurred at the LSC-PVC interface.

Numerical Example: Figure 10 shows the result of a numerical calculation of the light intensity arriving at the PVC of a PSC geometry device. This calculation is done by a finite element analysis which can be applied to an arbitrary LSC geometry and which is developed in Appendix C. The three plots are for matrix optical densities of $0,0.01$, and 0.15 as measured across the width of the PSC. The peak in the curves occurs at $47^{\circ}$, which is the compliment of the critical angle. It is striking to note that if the matrix material is completely clear, there is a substantial amount of light arriving at the PVC at a fully glancing angle of incidence. This effect is peculiar to the infinite geometry of the PSC and is a strong point against utilizing the PSC as a prototype LSC.

We define $I(\theta, \bar{\nu})$ to be the number of photons per unit area arriving at the LSC-PVC interface as a function of angle of incidence and wave number. In an arbitrary geometry this intensity will also be a function of the position along the edge. However, we will assume that the edge illumination is sufficiently uniform that the variation with position is ignorable. The total number of photons arriving at the PVC, defined to be $W$ [Eq. (49)], is then the area of the PVC times the integral of $I(\theta, \bar{\nu})$ over angle and wave number:

$$
W=A_{e} \int_{0}^{\infty} d \bar{\nu} \int_{0}^{\pi / 2} d \theta I(\theta, \bar{\nu})
$$

We are interested in averaged transmission and reflection coefficients $T_{\mathrm{pvc}}$ and $R_{\mathrm{pvc}}$ such that the total number of photons delivered into the PVC is given by $W T_{\text {pvc. }}$ The calculation of $T_{\text {pvc }}$ is slightly complicated by the likelihood that an antireflection coating will be used in the PVCs. One reason for their use is that antireflection coatings can be made quite effective for the relatively narrow band of wavelengths of the output light. Also the small area and good isolation from the environment make the cost and rate of deterioration of such coatings low. Using the Fresnel equations for transmission through an AR coating [Eqs. (8)], we find that the reflection coefficient $R_{\text {pvc }}$ at the LSC-PVC interface is given by

$$
\begin{aligned}
R_{\mathrm{pvc}} & =\int_{0}^{\infty} d \bar{\nu}\left[\int_{0}^{\pi / 2} d \theta R(\theta, \bar{\nu}) I(\theta, \bar{\nu})\right] \\
& / \int_{0}^{\infty} d \bar{\nu} \int_{0}^{\pi / 2} d \theta I(\theta, \bar{\nu}) .
\end{aligned}
$$

The transmission coefficient is then

$$
T_{\mathrm{pvc}}=1-R_{\mathrm{pvc}} .
$$

Finally we must take into account the response of the PVC to the light output of the LSC. From Wolf's paper on photovoltaic converters ${ }^{12}$ the energy efficiency of a $\mathrm{PVC}$ is given by

$$
\eta_{\mathrm{pvc}}=\left(\text { V.F.)(F.F.) } \frac{q \int_{E_{g / h \mathrm{c}}}^{\infty} P_{\mathrm{in}}(\bar{\nu}) d \bar{\nu} / \bar{\nu}}{h c \int_{0}^{\infty} P_{\mathrm{in}}(\bar{\nu}) d \bar{\nu}} E_{g},\right.
$$

where V.F. is the voltage factor, F.F. is the fill factor, $q$ is the electric charge, $E_{g}$ is the bandgap energy of the particular semiconductor, and $P_{\text {in }}(\bar{\nu})$ is the power input per wave number and unit area to the device. The approximations included in this equation are complete absorption of energies higher than $E_{g}$, complete collection of electron-hole pairs, and no series resistance losses. In fact these approximations are particularly good in the context of using PVCs in an LSC. The collected light will typically be of energies about $0.3 \mathrm{eV}$ above the bandgap of the PVC, so that especially in silicon the absorption will be deep enough to prevent large amounts of surface recombination. Because the actual exposed area of the cell is narrow (e.g., $2 \mathrm{~mm}$ ), contact blockage of light as well as surface resistance losses should be minimized.

The power input to the PVC per unit area is the incident flux times the energy per photon times the transmission coefficient:

$$
P_{\text {in }}(\bar{\nu})=h c \int_{0}^{\pi / 2} d \theta T(\theta, \bar{\nu}) I(\theta, \bar{\nu}) \bar{\nu} .
$$

Combining this result with Eq. (54)

$$
\eta_{\mathrm{pvc}}=(\mathrm{V} . \mathrm{F} .)(\mathrm{F} . \mathrm{F} .) \frac{q \int_{E_{s / h c}}^{\infty} d \bar{\nu} \int_{0}^{\pi / 2} d \theta T(\theta, \bar{\nu}) I(\theta, \bar{\nu})}{h c \int_{0}^{\infty} d \bar{\nu} \cdot \bar{\nu} \int_{0}^{\pi / 2} d \theta T(\theta, \bar{\nu}) I(\theta, \bar{\nu})} E_{g} .
$$

The fill factor F.F. is the ratio of the available power from a given cell to the product of the open circuit voltage and short circuit current for that cell. F.F. is relatively independent of the application of the device, having values staying near 0.82 for both silicon and gallium arsenide cells. ${ }^{6}$ The voltage factor V.F. is the ratio of the open circuit voltage to the energy gap of the semiconductor ${ }^{12}$ and has the units of inverse charge:

$$
\text { V.F. }=V_{\text {oc }} / E_{g}=\frac{A k T}{q E_{g}} \ln \left(\frac{I_{s c}}{I_{0}}+1\right) .
$$

$A$ is a perfection factor, $E_{g}$ is the band-gap energy, $I_{s c}$ is the short-circuit current, and $I_{0}$ is the saturation current for the cell. Various theories give different predictions for $A$ and $I_{0},{ }^{13-15}$ though they all agree on the voltage factor. If $A$ is taken to be $1, I_{0}$ is predicted to be

$$
I_{0}=e\left(\frac{L_{h} p_{n_{0}}}{\tau_{h}}+\frac{L_{e} n_{p_{0}}}{\tau_{e}}\right),
$$

where $L_{h}$ is the hole diffusion length, $\tau_{h}$ is the hole lifetime, $p_{n_{0}}$ is the equilibrium minority electron density, $L_{e}$ is the electron diffusion length, $\tau_{e}$ is the electron lifetime, and $n_{p_{0}}$ is the equilibrium minority hole density. ${ }^{16}$

While the main advantage of the LSC is to reduce the required acreage of PVCs, the high flux gains possible in an LSC will cause the cells that are used to have a higher effective efficiency due to the logarithmic de- 
pendence of V.F. on the flux delivered to the cell [Eq. (57)]. Because this high density flux is near the bandgap energy, thermal heating of the cells is minimized. For constant cell temperature, we have that the voltage factor for a cell used in an LSC is

$$
\text { V.F. }=\text { V.F.AM1 } \ln \left[\frac{\left(I_{\mathrm{sc}}\right)_{\mathrm{LSC}}-\left(I_{\mathrm{sc}}\right)_{\mathrm{AM} 1}}{I_{0}}\right],
$$

where V.F.AM1 and $\left(I_{\mathrm{sc}}\right)_{\mathrm{AM} 1}$ are the voltage factor and short circuit current, respectively, for the cell exposed to an air mass one solar spectrum, and $\left(I_{\mathrm{sc}}\right)_{\mathrm{LSC}}$ is the short circuit current for the cell in contact with an LSC. $\left(I_{\mathrm{Sc}}\right)_{\mathrm{LSC}}$ is the flux per unit area delivered to the cell, $W / A_{e}$, times the transmission coefficient $T_{\mathrm{pvc}}$ times the charge $q$ :

$$
\left(I_{\mathrm{sc}}\right)_{\mathrm{LSC}}=W T_{\mathrm{pvc}} q / A_{e} .
$$

All the necessary parameters for predicting the output of a single dye LSC have now been developed. In the next section we will combine these to create the final system performance parameters.

\section{System Performance Parameters- $G_{f}, \eta_{\text {Isc }}$}

For an ideal LSC, the flux that emerges from the edge is more intense than the solar flux by the ratio of the area of the face to the area of the edge, a quantity we have previously defined as the geometric gain $G_{\text {geom }}$. The actual ratio is of course smaller than $G_{\text {geom }}$ and is a good measure of the quality of an LSC as a collector of light. We therefore define the flux gain of the system $G_{f}$ as the increase in the usable photon flux delivered into the PVC when it is used with an LSC as opposed to when the PVC directly faces the sun. Mathematically $G_{f}$ is the flux per unit area transported to the PVC times the transmission coefficient for the LS-PVC interface divided by the fraction of the incident solar spectrum whose energy is above the band-gap energy times the air-PVC transmission coefficient $T_{0_{\mathrm{pvc}}}$ :

$$
G_{f}=\frac{W T_{\mathrm{pvc}}}{A_{e} T_{0_{\mathrm{pvc}}} \int_{E_{g / h c}}^{\infty} d \bar{\nu} N(\bar{\nu})}
$$

Using Eq. (49), which gives $W$ in terms of the loss coefficients $r, \bar{r}, P, \delta$, and $\eta$, the solar absorption per unit area $S$, and using the fact that $G_{\text {geom }}=A_{f} / A_{e}$ give the following:

$$
G_{f}=G_{\mathrm{geom}} \frac{T_{\mathrm{pvc}} S \eta(1-P)(1-r)(1-\delta)}{T_{0_{\mathrm{pvc}}}(1-\eta[\bar{r} P+r(1-P)]) \int_{E_{g / h c}}^{\infty} d \bar{\nu} N(\bar{\nu})}
$$

Another useful parameter is the total system efficiency, $\eta_{\text {lsc }}$, defined as the total electrical energy output divided by the total solar energy input. $\eta_{\mathrm{lsc}}$ is the energy of the collected light delivered to the PVC times the transmission into the PVC times the efficiency of conversion of the PVC divided by the solar energy incident on the LSC:

$$
\eta_{\mathrm{lsc}}=\frac{A_{e} \int_{E_{s / h c}}^{\infty} d \bar{\nu} \cdot \bar{\nu} \int_{0}^{\pi / 2} d \theta I(\theta, \bar{\nu}) T(\theta, \bar{\nu})}{A_{f} \int_{0}^{\infty} d \bar{\nu} N(\bar{\nu}) \cdot \bar{\nu}} \eta_{\mathrm{pvc}} \cdot
$$

If we insert the definition of $\eta_{\mathrm{pvc}}$ from Eq. (56) in Eq. (63), using the fact that the open circuit voltage $V_{\mathrm{oc}}=$ (V.F.) $E_{g}$, we have that

$$
\eta_{\mathrm{lsc}}=\frac{(\mathrm{F} . \mathrm{F} .) V_{\mathrm{oc}} q A_{e} \int_{E_{s / h c}}^{\infty} d \bar{\nu} \int_{0}^{\pi / 2} d \theta I(\theta, \bar{\nu}) T(\theta, \bar{\nu})}{h c A_{f} \cdot \int_{0}^{\infty} d \bar{\nu} \cdot \bar{\nu} N(\bar{\nu})} .
$$

Using Eqs. (51)-(53), this can be put in the form

$$
\eta_{\mathrm{Isc}}=\frac{V_{\mathrm{oc}} q(\mathrm{~F} . \mathrm{F} .) W T_{\mathrm{pvc}}}{h c A_{f} \cdot \int_{0}^{\infty} d \bar{\nu} \cdot \bar{\nu} N(\bar{\nu})} .
$$

If we now insert the definition of $W$ from Eq. (49), we have the final form of the LSC efficiency:

$$
\eta_{\mathrm{lsc}}=\frac{V_{\mathrm{oc}} q(\mathrm{~F} . \mathrm{F} .) T_{\mathrm{pvc}} \eta(1-P)(1-r)(1-\delta) S}{h c\{1-\eta[\bar{r} P+(1-P) r]\} \cdot \int_{0}^{\infty} d \bar{\nu} \cdot \bar{\nu} N(\bar{\nu})}
$$

Numerical Example: To demonstrate the use of these system parameters, we will calculate the flux gain and system efficiency of a particular single dye LSC-a PSC $10 \mathrm{~cm}$ across and $2 \mathrm{~mm}$ thick composed of PMMA doped with 0.001 moles/liter of rhodamine-6G with edge-mounted GaAs solar cells. The geometric gain is $G_{\text {geom }}=A_{f} / A_{e}=50$. Assume that the PVCs are AR coated, so that $T_{\mathrm{pvc}}=0.95$. The quantum efficiency of luminescence for rhodamine-6G is $\eta=0.95$. The integrated solar flux above the band-gap energy is $2.4 \times$ $10^{21}$ photons $/ \mathrm{m}^{2}$. $S$ for this system is $5.1 \times 10^{20}$ photons $/ \mathrm{m}^{2}$. From Fig. 8 the transmission loss is $\delta=0.08$, and the critical cone loss is $P=0.26$. The self-absorption coefficients are $r=0.65$ and $\bar{r}=0.4$. Inserting these values in Eq. (62), we find that the flux gain for this system is $G_{f}=5.3$. If we assume an open circuit voltage of $V_{\mathrm{oc}}=0.93$ and that the integral of the incident solar energy is $5.7 \times 10^{21} \mathrm{~V} / \mathrm{m}^{2} / \mathrm{sec}$ and using a fill factor of F.F. $=0.82$, we find the system efficiency is $\eta_{\text {lsc }}$ $=3.4 \%$.

If the above calculated performance was typical of the theoretically limiting values, the LSC would not be conspicuously promising as a solar technology. Only about $20 \%$ of the sunlight above the cell band gap is being utilized in this single dye device. However, if a multitude of dyes are used to more thoroughly cover the spectrum, this fraction can be increased by at least a factor of 3 . This technique will be explored in detail in a future publication.

\section{Dye Selection and Photochemistry}

Of the presently available luminescent absorbers, the fluorescent laser dyes appear to be the most promising for use in prototype LSCs. We will now examine dye concentration, orientation, and photobleaching, specifically as they pertain to the organic laser dye based LSC.

\section{A. Concentration and Spectral Position}

In a single dye LSC the dye concentration is determined by the dye's solubility in the matrix material, the portion of the solar spectrum that the dye is expected 
to absorb, and high concentration quenching effects. Typical organic laser dyes are polar, and the limit of their solubility in essentially nonpolar PMMA is $10^{-3}-10^{-2} M$. Good absorption of sunlight means that the optical density of the LSC should be at least 2 in the region of the spectrum where the device is expected to operate. In Fig. 5, the top left graph shows that a typical single dye in an LSC at a concentration of about 0.0005 moles/liter gives good absorption across a 2000 -wave number region. Increasing the concentration by a factor of 10 increases this region to about 5000 wave numbers. In a single dye system the only method to increase the total solar absorption is to increase the dye concentration as much as possible. Though increasing the dye concentration increases the rate of self-absorption, the lower graph of Fig. 6 shows that the collected flux will always increase for increasing dye concentrations. When the dye concentration is high enough, each dye molecule becomes perturbed by the fields from neighboring dye molecules resulting in expected decreases in quantum efficiency, or quenching of the luminescence.

\section{B. Dye Molecule Orientation}

Each time a photon is radiatively emitted in the LSC, the photon has a chance to be lost out of the faces of the LSC via the critical cones. One technique to reduce this problem is to modify physically the geometry of the LSC so as to reduce the actual size of the critical cones, as will be discussed in a future publication. However, another possible technique is to utilize the fact that the dye molecules typically absorb and emit as electric dipoles, such that if these dipoles are oriented perpendicularly to the plane of the LSC, the toroidal emission pattern of the dipole will help to restrict the emitted flux to the region outside of the critical cones.

Assume that all the dye emission dipoles are oriented parallel to the normal of the LSC plane. The dipole emission intensity varies as $\sin ^{2}(\theta)$, so that the new probability $P$ for escape out of the critical cones for this nonisotropic system is given by

$$
P=\frac{2 \int_{0}^{\theta_{c}} \sin ^{3}(\theta) d \theta}{\int_{0}^{\pi} \sin ^{3}(\theta) d \theta} .
$$

Integrating, we find that

$$
\begin{aligned}
P & =1-3 \cos \left(\theta_{c}\right) / 2+\cos ^{3}\left(\theta_{c}\right) / 2 \\
& =1-\left(1+1 / 2 n^{2}\right)\left(1-1 / n^{2}\right)^{1 / 2},
\end{aligned}
$$

where $n$ is the index of refraction of the matrix. For $n$ $=1.49$, we find that $P=0.092$, as opposed to 0.259 in the case of random dye orientation.

\section{Photobleaching and Thermal-Bleaching Effects}

Perhaps the most critical outstanding question in the implementation of the LSC technology is the stability and lifetime of the dyes under the conditions found in an exposed environment. Though the process is not presently well understood, there are two basic types of deterioration.
Thermal bleaching, or the breakdown of the dyes by heating, has been studied in solutions of rhodamine$6 \mathrm{G}^{17}$ and has been found to obey a highly nonlinear temperature dependence. At $60^{\circ} \mathrm{C}$ no dye instability was seen in the solutions tested, so that both in the casting process and under solar illumination we anticipate the effects of thermal bleaching to be negligible.

The second process is loosely termed photobleaching, meaning any process at a moderate temperature whereby the absorption of light by the sample leads to deterioration of the dye. Typically it is assumed that within the individual wavelength regions of visible, near, middle, and far UV, that the bleaching rate is wavelength independent. Experiments involving excitation Iiom an xenon lamp have shown a bleaching rate of about one dye molecule per $10^{6}$ excitations in a solution. ${ }^{18,19}$ We have found however that this deterioration rate seriously overestimates the actual rate that we have measured for plastic matrices doped with dyes.

In order to begin to resolve the question of the environmental stability of LSCs consisting of laser dyes dissolved in a plastic matrix, we have begun a series of tests of dye stability in PMMA samples. The results reported here are preliminary and represent only lower bounds on the dye stability.

An LSC plate containing about $10^{-4}$ moles/liter of the dyes rhodamine- $6 \mathrm{G}$ and coumarin- 6 was obtained from Acrilex, Inc. ${ }^{20}$ Samples of this material $0.3 \mathrm{~cm} \times 1 \mathrm{~cm}$ $\times 3 \mathrm{~cm}$ in size were cut from the plate. [Note on composition: $0.34 \mathrm{~g}$ of rhodamine-6G tetrafluoborate $(=$ $0.0485 \mathrm{~g} /$ liter $)$ and $0.24 \mathrm{~g}$ of coumarin $-6(=0.0343 \mathrm{~g} / \mathrm{liter})$ were dissolved in 7 liters of monomer. These concentrations are in the range used by Swartz et al. ${ }^{1}$ in previous work on multiple dye LSCs.] Optical absorption spectra were measured, and the optical density of the spectral peaks of rhodamine-6G and coumarin- 6 at 530 $\mathrm{nm}$ and $460 \mathrm{~nm}$, respectively, were noted for the asreceived material.

The LSC samples were then placed in a QUV accelerated weathering tester ${ }^{21}$ and subjected to a test cycle consisting of $8 \mathrm{~h}$ of exposure to UV light from a bank of four S-40 UV fluorescent lamps at a temperature of $60^{\circ} \mathrm{C}$ followed by $4 \mathrm{~h}$ of darkness at $50^{\circ} \mathrm{C}$ and $100 \%$ relative humidity. This test cycle was repeated for a total of $2400 \mathrm{~h}$. The samples were removed from the QUV at approximately 200 -h intervals at which times their optical absorption spectra were measured. ESR spectra were also taken during some of these measurement intervals.

The fraction of the dye remaining at a given time was estimated by taking the ratios of the optical densities at the absorption peaks of the exposed dyes to the samples prior to QUV exposure. Figure 11 shows a plot of the peak optical density for rhodamine-6G and coumarin-6 as a function of total test hours.

The data show that there are at least two distinct periods in the depletion of the dyes. During an initial period of 200-600 h, the dye concentration follows an exponential decay, $C_{0} \exp \left(-t / t_{1}\right)$, where $t_{1}=760 \mathrm{~h}$ and $1000 \mathrm{~h}$ for rhodamine-6G and coumarin-6, respectively. For coumarin-6, this initial period is followed by a 


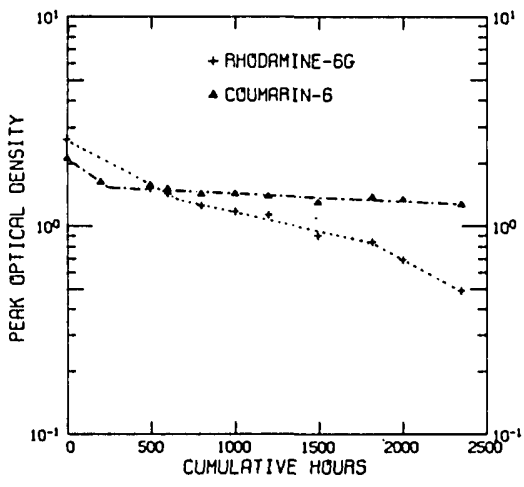

Fig. 11. Here we plot the experimentally measured deterioration of the laser dyes rhodamine- $6 \mathrm{G}$ and coumarin- 6 under xenon lamp illumination in a QUV 21 test chamber. Typically an hour of exposure in such a test chamber represents between $8 \mathrm{~h}$ and $25 \mathrm{~h}$ of exposure in normal environmental conditions. After an initial period of rapid deterioration, both dyes reached slower rates of deterioration, which the coumarin dye then maintained for the duration of the test.

change in the dye decay time constant to $t_{2}^{\prime}=10,000 \mathrm{~h}$. Rhodamine-6G on the other hand changes its decay time to $t_{1}^{\prime}=2300 \mathrm{~h}$ for the period from about $600 \mathrm{~h}$ to $1800 \mathrm{~h}$, after which the decay time decreases to approximately its initial value. While no free radicals were detected in the received materials, they were detected in the first and second periods of the test, with the concentrations being higher in the first part of the test. Identification of the nature of the radicals involved has not yet been made.

The main question of practical interest is: what can these preliminary tests tell us about the environment durability of LSCs based on organic dyes in a PMMA matrix? It is important to point out that the QUV instrument does not reproduce actual environmental conditions. It provides an accelerated test that is likely to be more severe for organic molecules than common terrestrial conditions. For example, the UV flux present within the test apparatus has a peak intensity at $313 \mathrm{~nm}$. The intensity at that wavelength is $18 \mu \mathrm{W}$ per sq. $\mathrm{cm} / \mathrm{nm}$. The temperatures used in the cycle are higher than average ambient, and the 100\% humidity conditions are also very severe. The real environmental hours represented by an hour of QUV test will vary with the composition of the LSC and with the particular design of the system. However, factors in the range of 8-25-h environmental exposure per hour of QUV test time have been suggested.22 Thus the evidence of the test points to a time for $50 \%$ loss of the dye population of at least $700 \mathrm{~h}$ ( 0.08 years) for rhodamine- $6 \mathrm{G}$ and 2400 $\mathrm{h}$ (0.27 years) for coumarin-6 in PMMA if no acceleration factor is assumed, and to a $50 \%$ loss time of $1.75 \times$ $10^{4} \mathrm{~h}\left(2\right.$ years) and $6 \times 10^{4} \mathrm{~h}$ (6.8 years) for these dyes, respectively, using the largest acceleration factor. It should be noted that no measures were taken to enhance the durability of the dyes. We expect that relatively inexpensive protection measures such as a top cover glass and bottom aluminum reflector or the addition of antioxidants or UV absorbers, as well as development and application of polymer technology, will substantially lengthen the operating life of the organic dye LSC. Also, inorganic systems may be used in the future.

\section{Modified PVCs for Use with an LSC}

Solar cells being developed today are typically designed to convert the entire solar spectrum into electricity as efficiently as possible. This requirement in turn dictates the choice of bandgap, junction depth, optical coating, and so on. However, the requirements of a PVC when mounted on an LSC are quite different, and suitable variations of the present technology should be developed.

Because all the incident flux can be assumed to be at an energy just above the bandgap energy, absorption will take place at relatively large depths in the cell. For direct bandgap materials this probably will not have an appreciable effect, but for the indirect materials this means that the depletion region can be made quite thick, eliminating much of the resistive loss in the cell.

Since the LSC plate can be made relatively thin (e.g., $2 \mathrm{~mm}$ ), there should be no need for opaque contacts over the active region of the cell. This will immediately increase the effective cell efficiency.

As discussed by Goetzberger, ${ }^{23}$ the most important development in this area will be a high efficiency cell with a bandgap in the region of $2-2.6 \mathrm{eV}$.

Finally the optical coating is particularly important in this application because of the oblique angles of incidence typical of light fluxes from the LSC. An interesting possibility is to use roughened surface cells, such as the Comsat nonreflecting (CNR) design, ${ }^{24}$ to cut down on reflective losses at the LSC-PVC interface.

\section{Summary of Results}

There are three main results or issues that we would like to emphasize here in summary form. Following these points we will present a highly simplified model distilled from the preceding calculation that gives a rough but rapid estimation of the performance of a particular LSC design.

(1) In this paper we have developed a photon flow model that describes the operation and predicts the performance of a single dye LSC. The lumped parameters in the model are derived analytically from spectroscopic measurements of the component dye and matrix material.

(2) Because the absorption band of a dye or inorganic ion overlaps with the emission spectrum, it is always possible for reabsorption or self-absorption of luminescence by the luminescing species to take place. We find that in the long path lengths and high absorber concentrations typical of an LSC, self-absorption is a dominant effect, generating losses by giving a photon several opportunities to be internally converted into phonons or to be emitted into the critical escape cones. We have developed a formalism that predicts the magnitude of the self-absorption effect and have offered several techniques to minimize the resulting losses. 
(3) We have begun investigations of the tendency of organic laser dyes in plastic hosts to degrade under environmental exposure. Although the evidence is as yet sparse, we draw the following tentative conclusions:

Under conditions of this test the time to 50\% degradation of at least one laser dye in a PMMA matrix is over $2400 \mathrm{~h}$ and may be longer than $60,000 \mathrm{~h}$ (6.8 years) under actual environmental conditions.

Different dyes decay at different rates and presumably by different chemical pathways in the same matrix material.

Free radicals occur in dye-PMMA LSCs under these accelerated conditions, with the highest radical concentrations occurring during periods of rapid dye loss.

Dye chemistry under actual and simulated operating conditions should be pursued as an important part of future LSC research. In particular, the dye quantum efficiency as a function of test hours, which was not measured in our tests, should be investigated.

In general to obtain accurate predictions of the performance of a particular LSC, one should proceed through the calculations as prescribed in Sec. II. However, there are occasions when a quick calculation is desirable, and we have found that some simple relations can often give surprisingly good results. We make the approximation that the only loss mechanisms that are appreciable are the quantum efficiency of the dye, luminescence escape out of the critical cones, and selfabsorption. From Eq. (18) we see that in this approximation if a photon interacts with just one dye molecule in the LSC plate, the probability that the photon will be trapped and transported to the PV.C is the quantum efficiency times the probability that the luminescence will occur outside of the critical cones: $\eta(1-P)$. (This assumes that there is no self-absorption in the critical cones.) However each photon will typically undergo more than one emission before reaching the PVC; in fact in Sect. II.E we calculated the average number of emissions $N_{e}$ that a photon undergoes before reaching the PVC. Each emission will be trapped with a probability $\eta(1-P)$, so that the total probability of trapping and collecting the photon* is $[\eta(1-P)]^{N_{e}}$. The absorbed solar flux per unit area divided by the total integrated solar flux per unit area, $S / I$, is the fraction of the total solar flux that is absorbed by the LSC. The fraction of the total solar flux that is incident on the edge-mounted PVC is $[\eta(1-P)]^{N_{e} S / I}$. Finally we want to only consider that part of the solar flux $\xi$ which is higher in energy than the absorption cutoff for the particular cell:

$$
\xi=\int_{E_{g / h c}}^{\infty} N(\bar{\nu}) d \bar{\nu} / \int_{0}^{\infty} N(\bar{\nu}) d \bar{\nu},
$$

where $N(\bar{\nu})$ is the solar flux per wave number, $\xi$ (silicon) $=0.64$, and $\xi$ (gallium arsenide) $=0.42$. The fraction of the solar flux above the bandgap of a particular semiconductor that emerges from the LSC is $[\eta(1-$ $P)]^{N_{e}} S / I \xi$. In this approximation, the flux gain $G_{f}$ from Eq. (62) becomes this fraction of the usable solar flux that is collected times the geometric gain of the collector:

$$
G_{f}=G_{\text {geom }}[\eta(1-P)]^{N_{e}} S / I \xi
$$

The system efficiency $\eta_{\text {lsc }}$ from Eq. (66) becomes the efficiency of the cell exposed directly to the full solar spectrum, $\eta_{\mathrm{pvc}}$, times the fraction of the usable solar flux that is delivered to the cell:

$$
\eta_{\mathrm{lsc}}=\eta_{\mathrm{pvc}}[\eta(1-P)]^{N_{e} S / I \xi}
$$

Of course, to be valid, the emission from the dye must be above the energy of the absorption edge of the PVC used.

Numerical Example: For a typical single dye LSC, $\eta=0.9,(1-P)=0.74, N_{e}=2.0, G_{\text {geom }}=100.0$, and $S / I$ $=0.15$ [Eq. (13)]. The fraction of the total solar flux that is collected at the edge is $[\eta(1-P)]^{N_{e} S / I}=0.0665$. We assume total solar spectrum conversion efficiencies of $18 \%$ and $21 \%$ for silicon and gallium arsenide cells, respectively. Silicon utilizes about $\xi=64 \%$ of the spectrum, while gallium arsenide uses about $42 \%$. We find that a silicon cell attached to this LSC will see a flux gain of 10.4 and have a system efficiency of $1.9 \%$, while a gallium arsenide cell will see a flux gain of 15.8 and have a system efficiency of $3.3 \%$.

Equations (70) and (71) incorporate a number of approximations, so that their use requires some discrimination. We have assumed that the entire luminescence spectrum is above the absorption edge of the solar cell. We have also assumed no scattering, reflective losses, or matrix absorption takes place during transport to the cell. Finally, the number of emissions $N_{e}$ is that derived in Eq. (22) only in the limit that self-absorption in the critical cone is negligible. An accurate calculation of the flux gain and system efficiency will require the use of the formalism outlined in Sec. II.

Note added in proof: Recent experiments performed here have shown that the approximation of truncating the emission spectra, used in Section II.E, is valid especially in the low temperature limit. More on this will be published elsewhere.

A. H. Zewail is an Alfred P. Sloan Fellow. Part of this work was done while T. Cole was a Sherman Fairchild Distinguished Scholar at Caltech. This work was supported in part by a contract from the U.S. Department of Energy and in part by ARCO Solar, Inc. We thank L. Freeman, D. L. Garrett, and H. Van One of the Polymer Sciences Department, Ford Engineering and Research Staff, for performing the accelerated weathering tests and for their generous help in the analysis and interpretation of the results. We also thank J. McCaldin of the Applied Physics Department at Caltech for stimulating discussions.

This is Contribution 6005 from the California Institute of Technology.

\section{Appendix A. Previous Development}

The first publication in the open literature proposing the use of light pipe trapping of luminescence as a means to enhance photovoltaic solar energy conversion was that of Weber and Lambe. ${ }^{10}$ Somewhat earlier Lerner ${ }^{25}$ had built a solar collecting device embodying this principle, consisting of a solution of laser dye contained between two sheets of glass. (This device was described in a 1973 NSF proposal and was rejected.) In 1975 D. P. Weilmenster, working under Lerner's direction at MIT, submitted a senior thesis entitled 
"Radiation Transfer Process in Rhodamine-6G Methanol Applied to Solar Energy Conversion." None of the work at MIT saw publication in the open literature.

New developments and ideas have appeared in recent publications. Levitt and Weber ${ }^{26}$ gave results on $\mathrm{Nd}$ doped glass and rhodamine-6G in PMMA as prototype PSCs. Goetzberger and Grubel ${ }^{5}$ described an LSC using a stack of dye doped plastic, with each sheet matched to a particular type of photovoltaic cell. Swartz et al. ${ }^{1}$ demonstrated energy transfer in multiple-dye plastic matrices as a method of achieving more efficient solar energy collection. Bauer and Grubel ${ }^{27}$ have suggested using the LSC principle to make liquid crystal displays, and Goetzberger ${ }^{23}$ observed the promise of using LSCs to concentrate diffuse sunlight.

The idea of light trapping to achieve enhanced radiant intensity is now new. A device for radiance amplification in scintillation counters was proposed by Shurcliff ${ }^{28}$ in 1951 based on the calculation of trapping efficiency by Shurcliff and Jones. ${ }^{29}$ Gárwin ${ }^{30}$ discussed the thermodynamic aspects of trapped radiation converters, and $\mathrm{Kiel}^{31}$ published results of experiments on plastic radiation converters for scintillation counters. The LSC concept has also been used for astronomical observations by Weekes. ${ }^{32}$ Sheets of dye doped PMMA were edge-coupled to photomultiplier tubes and used in an attempt to observe Cerenkov radiation from cosmic ray showers in the upper atmosphere.

\section{Appendix B. Effect of Multiple Internal Reflections Within the Critical Cones}

Throughout the text we have assumed that light within the LSC which is incident on one of the LSC faces at an angle of incidence less than the critical angle, i.e., light within the critical cones, is completely transmitted into the surrounding air. This assumption implies that the incident sunlight passes once through the LSC plate and then is lost and that if self-absorption effects are ignored that any luminescence inside the critical cones is also lost.

To check the validity of this assumption we will now explicitly calculate the effect of internal reflections within the critical cones on $P$, the probability that isotropic luminescence will be lost out of the critical cones, and on $S$, the absorbed solar flux per unit area. For this

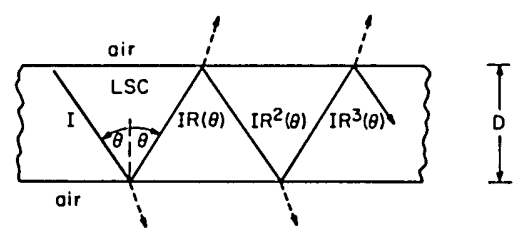

Fig. 12. The geometry used in calculating the effect of Fresnel reflections inside of the critical cones. We begin with a ray of intensity $I$ in a flat LSC plate of thickness $D$. This ray is incident to the surface at an angle 0 , and the fraction of the ray that is reflected is $R(\theta)$. calculation we will use the planar geometry of Fig. 12 for an LSC plate of thickness $D$ surrounded by air. Assume that light of intensity $I$ originates near the surface of the LSC and propagates so as to intercept the opposite surface with an angle of incidence $\theta$. Due to the index mismatch at the air-LSC interface, there will be partial internal reflection of magnitude $R(\theta)$ given by the Fresnel equations [Eq. (5)], so that the intensity after the first reflection is $\operatorname{IR}(\theta)$, after the second reflection is $I R^{2}(\theta)$, and so on.

The first question we ask is: for what values of $\theta<$ $\theta_{c}$ does the intensity propagate far enough in the LSC that it will contribute to the light absorbed by the PVC? To find out we define $I(x)$ to be the intensity inside the LSC at a horizontal distance $x$ from the point of emission:

$$
I(x)= \begin{cases}I & 0 \leq x<D \tan (\theta) \\ I R(\theta) & D \tan (\theta) \leq x<2 D \tan (\theta) \\ I R^{2}(\theta) & 2 D \tan (\theta) \leq x<3 D \tan (\theta) \\ \quad \vdots & \vdots\end{cases}
$$

For large $x$ we can approximate this expression by the following:

$$
I(x)=I R^{x / D}(\theta)=I \exp \{x \ln [R(\theta)] / D\} .
$$

If we now assert that luminescence must lose no more than half of its intensity in traveling a distance 10 times the thickness of the LSC in order to be considered trapped, we have the criteria that all luminescence is trapped whose angle of incidence to the faces is greater than or equal to $\theta^{\prime}$, where $\theta$ is given by

$$
1 / 2=\exp \left\{10 \ln \left[R\left(\theta^{\prime}\right)\right]\right\} .
$$

For an index of refraction of 1.49 , the above requirement says that all light is trapped whose angle of incidence is greater or equal to $\theta^{\prime}=42.1515^{\circ}$. Since the critical angle for this material is $\theta_{c}=42.1552^{\circ}$, we find that including multiple internal reflections inside the critical cones decreases $P$ from 0.25867 to 0.25863 , which is completely negligible.

The second question of interest is to find how much internal reflection inside of the critical cones increases the absorbed solar flux $S$. The single pass LSC thickness traversed by the sunlight is $D / \cos (\theta)$. We now define a new effective single pass thickness $D^{\prime} / \cos (\theta)$, which is equal to the total effective path length over which absorption takes place:

$$
\left.\begin{array}{l}
D^{\prime}=D+D R(\theta)+D R^{2}(\theta)+D R^{3}(\theta)+\ldots \\
D^{\prime}=D /[1-R(\theta)]=D / T(\theta)
\end{array}\right\},
$$

where $T(\theta)$ is the transmission coefficient at the LSC-air interface. For an effective path length $D^{\prime}$ I $\cos (\theta)$ to be $10 \%$ longer than $D / \cos (\theta), \theta>36^{\circ}$, which means that the angle of incidence of the sunlight on the LSC must be greater than $61^{\circ}$. The effect is actually appreciable and will cause the LSC to perform slightly better at large angles of solar incidence than our uncorrected model would predict. The effect is slight because the solar absorption $S$ typically varies approximately as the logarithm of the absorption path length, as can be seen in Fig. 4. 
Appendix C. Finite Element Calculation of $I(\theta)$ and $\delta$

The collected flux incident on the PVC as a function of incident angle $I(\theta)$ and the average transport loss $\delta$ in general cannot be calculated analytically. The two entities are related; if $I^{\prime}(\theta)$ is the collected flux in the absence of matrix absorption losses and internal reflection losses, $\delta$ can be found by the following relation:

$$
\delta=1-\int_{0}^{\pi / 2} I(\theta) d \theta / \int_{0}^{\pi / 2} I^{\prime}(\theta) d \theta
$$
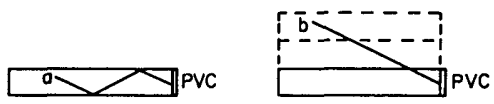

Fig. 13. (a) A typical photon trajectory in a planar LSC; (b) this trajectory can be unfolded by the method of images to form a straight line from the point of emission to the PVC.

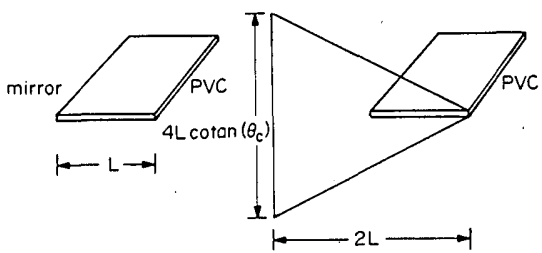

Fig. 14. This figure shows how the PSC geometry is unfolded from its original form on the left to the symmetric wedge form on the right. Any trajectory in the original geometry which is outside of the critical cones will intercept the PVC after some number of reflections, and for that trajectory there is a corresponding trajectory on the right which intersects the PVC at the same angle and has the same path length, but lies in a straight line.

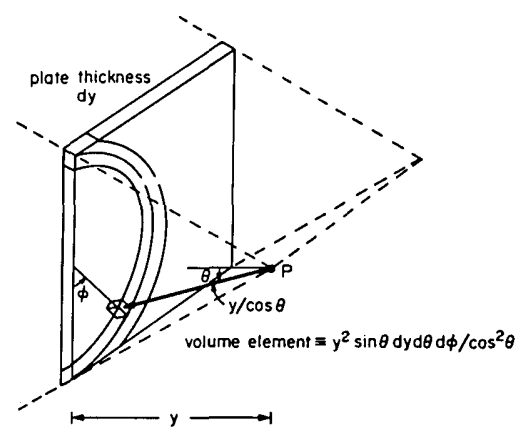

Fig. 15. This figure shows how the symmetric wedge of Fig. 14 is divided into finite elements. First the wedge is separated into plates of thickness $d y$, each plate being parallel to the PVC. Then each plate is broken into a series of concentric rings, each ring being $y d \theta / \cos (\theta)$ across, and the rings are subdivided into sections $y d \phi / \cos (\theta)$ wide, giving the finite element volume $y^{2} d y d \theta d \phi / \cos ^{2}(\theta)$.

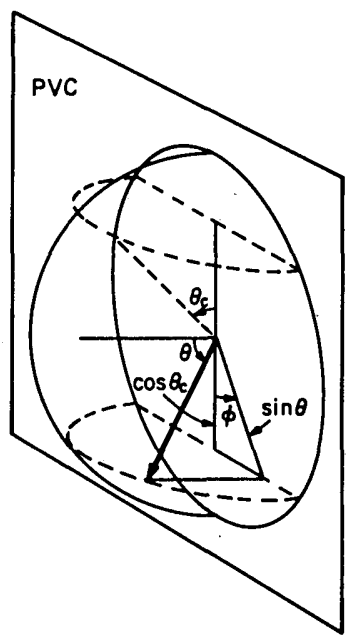

Fig. 16. The polar coordinate system in the frame of the PVC. The dotted semicircles represent sections of the half-sphere where there will be no incident light (because it has been lost out of the critical cones). For $\theta<\pi / 2-\theta_{c}$ there are no restrictions on the allowed values of $\phi$. For larger values of $\theta$ we find that $\phi$ cannot have values between $-\cos ^{-1}\left[\cos \left(\theta_{c}\right) / \sin (\theta)\right]$ and $\cos ^{-1}\left[\cos \left(\theta_{c}\right) / \sin (\theta)\right]$ and also between $\pi-\cos ^{-1}\left[\cos \left(\theta_{c}\right) / \sin (\theta)\right]$ and $\pi+\cos ^{-1}\left[\cos \left(\theta_{c}\right) / \sin (\theta)\right]$.

So the problem is reduced to finding $I(\theta)$ and $I^{\prime}(\theta)$ for the geometry of interest.

There are four steps in computing $I(\theta)$ for an arbitrary LSC geometry. The first is to unfold the geometry by the method of images to straighten the zigzag optical paths, formed by the multiple reflections off of the LSC faces, into rays eminating from a point on the PVC. Figure 13(a) shows the usual trapped photon propagating toward the PVC. In Fig. 13(b) we show the same photon propagation in the unfolded version, with the results that the optical path has been straightened, and the point of emission has been moved from point (a) to point (b). The second step is to divide the unfolded geometry into a number of finite elements such that each element subtends a constant solid angle and normal thickness with respect to the point of absorption on the PVC. This simplifies the integration by insuring that each element will contribute the same initial flux in the direction of the PVC. The third step is to find the limits of integration in the polar coordinates of the point on the PVC dictated by the position of these finite elements in the unfolded geometry. Finally we integrate over the contribution from every element multiplied by the Beers-Lambert attenuation $\exp [-\alpha(\bar{\nu}) r]$, where $r$ is the radial distance from the PVC to the point of emission, and $\alpha(\bar{v})$ is the matrix absorption coefficient over the dye emission spectrum. We will assume that total internal reflection losses due to surface roughness can be approximated by increasing $\alpha(\bar{\nu})$ appropriately. $I^{\prime}(\theta)$ is computed by doing the above integration without the exponential decay term.

It is instructive to demonstrate these steps in calculating $I(\theta)$ and $\delta$ for a specific LSC geometry, which in 
this case will be the PSC geometry of Fig. 1. As shown in Fig. 14, the unfolded PSC geometry is a symmetric wedge of width $2 L$ and minimum height $4 L \cot \left(\theta_{c}\right)$. The next step is to divide this unfolded geometry into a series of ribbons parallel to the PVC of thickness $d y$. These are then divided into circular sections of width $y d \theta / \cos (\theta)$, where $\theta$ is the polar angle from the point of absorption on the PVC. Such a finite element is pictured in Fig. 15 and satisfies the criteria of constant initial emission into a constant solid angle from the absorber over all the finite elements. The third step is to find the limits of $\theta$ and $\phi$ in the polar system about the absorption point on the PVC. By inspection of Fig. 16, $0 \leq \phi \leq 2 \pi$ for $0 \leq \theta \leq \pi / 2-\theta_{c}$, and $\phi$ runs between $\cos ^{-1}\left[\cos \left(\theta_{c}\right) / \sin (\theta)\right]$ and $\pi-\cos ^{-1}\left[\cos \left(\theta_{c}\right) / \sin (\theta)\right]$ and similarly between $\pi+\cos ^{-1}\left[\cos \left(\theta_{c}\right) / \sin (\theta)\right]$ and $2 \pi-$ $\cos ^{-1}\left[\cos \left(\theta_{c}\right) / \sin (\theta)\right]$ for $\pi / 2-\theta_{c} \leq \theta \leq \pi / 2$. Since they are symmetric, these limits can be simplified to yield the following integral form for $I(\theta)$ :

$$
\begin{aligned}
& I(\theta)=\left\{\begin{array}{l}
\int_{0}^{2 L} d y \int_{0}^{2 \pi} d \phi \sin (\theta) \exp [-y \alpha / \cos (\theta)] / 2 \pi \\
0 \leq \theta \leq \pi / 2-\theta_{c}
\end{array}\right. \\
& \left.-4 \int_{0}^{\cos ^{-1}\left(\cos \theta_{c} / \sin \theta\right)} d \phi \sin (\theta) \exp [-y \alpha / \cos (\theta)] / 2 \pi\right\} \text {, }
\end{aligned}
$$

where $\alpha$ is the absorption coefficient. $I^{\prime}(\theta)$ is given by Eq. (C2) in the limit that $\alpha=0$. The reason that the $y^{2} / \cos ^{2}(\theta)$ from the volume element does not appear in the integral is the $1 / r^{2}$ attenuation of the light that takes place in transit. The above equation for $I(\theta)$ simplifies to the following:

$$
\begin{aligned}
& I(\theta)= \\
& \left\{\begin{array}{r}
\int_{0}^{2 L} d y \sin (\theta) \exp [-y \alpha / \cos (\theta)] \quad 0 \leq 0 \leq \pi / 2-\theta_{c} \\
\int_{0}^{2 L} d y \sin (\theta) \exp [-y \alpha / \cos (\theta)] \mid 1-\frac{2}{\pi} \cos ^{-1}\left[\cos \left(\theta_{c}\right) / \sin (\theta)\right] \\
\pi / 2-\theta_{c} \leq \theta \leq \pi / 2
\end{array}\right.
\end{aligned}
$$

Omitting the exponential leaves an expression for $I^{\prime}(\theta)$. Combining these forms for $I(\theta)$ and $I^{\prime}(\theta)$ in Eq. (C1) gives the value for the transport loss $\delta$.

\section{Appendix D. Approximate Self-Absorption Calculation}

The usual approximations that are made in selfabsorption calculations are that a photon can be selfabsorbed at most once and that the energy distribution of the luminesced photons is given by the entire luminescence spectrum. In this approximation, the probability of self-absorption $r$ is given by ${ }^{9}$

$$
r=\int_{0}^{\infty} f(\bar{\nu})\{1-\exp [-\alpha(\bar{\nu}) l]\} d \bar{\nu},
$$

where $l$ is the path length over which self-absorption can occur, $f(\bar{\nu})$ is the normalized luminescence spectrum, and $\alpha(\bar{\nu})$ is the absorption coefficient.
In the limit of the above approximations, the selfabsorption formalism developed in Sec. II.E can be shown to give the same result. The collection efficiency for an LSC is given by Eq. (23):

$$
\begin{aligned}
Q= & (1-P)(1-r) \eta+(1-P)(1-r)[\bar{r} P+(1-P) r] \eta^{2} \\
& +(1-P)(1-r)[\bar{r} P+(1-P) r]^{2} \eta^{3}+\ldots
\end{aligned}
$$

If only the term corresponding to the first generation is retained, this becomes

$$
Q^{(1)}=(1-P)(1-r) \eta .
$$

For simplicity we will consider a spherical geometry, with luminescence occurring at the center of a sphere of LSC material of radius $l$, and with the entire outer surface of the sphere being covered by solar cells. In this case, the first generation collection efficiency of Eq. (30) simplifies to

$$
Q^{(1)}=\eta \int_{0}^{\infty} f(\bar{\nu}) \exp [-\alpha(\bar{\nu}) l] d \bar{\nu} .
$$

In this geometry, the probability of escape out of the critical cones $P$ is zero, so that equating Eqs. (D2) and (D3) gives

$$
(1-r)=\int_{0}^{\infty} f(\bar{\nu}) \exp [-\alpha(\bar{\nu}) l] d \bar{\nu} .
$$

Using the fact that $f(\bar{\nu})$ is normalized,

$$
\int_{0}^{\infty} f(\bar{\nu}) d \bar{\nu}=1,
$$

we find that Eq. (D1) is equivalent to Eq. (D4).

This approximation is good in cases where the concentrations are low and the path lengths over which self-absorption can take place are small, as shown by recent experiments. However, these approximations are typically not applicable to an LSC system.

It is revealing to calculate $N_{e}$, the number of emissions in an LSC experienced by a photon that arrives at the absorber, in this spherical geometry. Assume that the collection efficiency of the $i$ th generation $Q^{(i)}$ is given by the $i$ th power of the quantum efficiency times an arbitrary coefficient $l_{i}$ :

$$
Q^{(i)}=l_{i} \eta^{i}
$$

The only restriction on $l_{i}$ is that it is independent of $\eta$. Then the total collection efficiency is $Q$, where

$$
Q=\sum_{i} Q^{(i)}=\sum_{i} l_{i} \eta^{i}
$$

Taking derivatives with respect to $\eta$ and then multiplying by $\eta$ give

$$
\eta \frac{\partial Q}{\partial \eta}=\sum_{i} i l_{i} \eta^{i}=\sum_{i} i Q^{(i)} .
$$

From the definition of $N_{e}$ [Eq. (22)], we have that

$$
N_{e} Q=\sum_{i} i Q^{(i)}
$$

Combining the above results, we have

$$
\eta \frac{\partial Q}{\partial \eta}=N_{e} Q, \quad Q=(\eta)^{N_{e}} .
$$


In the more general case, where the critical cone loss is not zero, but where self-absorption in the critical cone is negligible, a similar procedure shows that

$$
Q=[\eta(1-P)]^{N_{e}} .
$$

This is the result used in the abbreviated calculation given in Sec. V.

The self-absorption probability, including all generations in this spherical geometry $r$, is found from Eq. (24) to be

$$
r=\frac{\eta-Q}{\eta(1-Q)} .
$$

Combining Eqs. (D9) and (D11), we see that

$$
N_{e}=1+\frac{\ln (1-r)-\ln (1-\eta r)}{\ln (\eta)} .
$$

For $\eta$ nearly 1 and for $r$ small, we have

$$
N_{e}=1+r .
$$

So in the low self-absorption limit, $N_{e}$ is one plus the probability of self-absorption. As $Q$ decreases, both $N_{e}$ and $r$ increase. As $r$ approaches one, $N_{e}$ increases much faster than $r$.

\section{Appendix E. Definitions}

AM1 spectrum The peak power solar spectrum at the earth's surface at vertical incidence on a clear day;

critical angle the minimum angle of incidence for which total internal reflection can take place;

critical cone one of at least two cones (usually one pointing upward and one downward) in an LSC characterized by the critical angle, such that all emission outside of these cones are totally internally reflected, and all emission inside can escape;

LSC luminescent solar concentrator, the general classification of collectors that utilize luminescence to concentrate sunlight;

matrix the rigid transparent material which supports the dye molecules or ions, typical matrix materials are glass and plastic;

PSC Planar solar concentrator, which is a specific geometry of LSC, shown in Fig. 1, it is an infinite flat ribbon, mirrored on the back and on one edge, with PVCs mounted on the remaining edge;

PMMA polymethyl methacrylate (e.g. Plexiglas);

photovoltaic cells.

PVC

Appendix F. Glossary of Symbols

$A_{e}$

Area of the edge of an LSC, or more generally the active area of absorber in an LSC;
C

$D$

$E_{b g}$

$f(\bar{\nu})$

F.F.

$G_{f}$

$G_{\text {geom }}$

$h$

$\hbar$

$I$

$I(\theta)$

$I(\theta, \bar{\nu})$

$I_{0}$

$I_{\mathrm{sc}}$

$J$

$L$

$l_{s}$

$n$

$N$

$N(\bar{\nu})$

$n_{1}$

$n_{2}$

$N_{e}$

$P$

$P_{\text {in }}(\bar{\nu})$ area of the face of an LSC, or more generally the surface area exposed to direct sunlight;

the concentration of dye or inorganic ion in the LSC matrix material, given in moles per liter;

the thickness of a PSC plate;

the bandgap energy of a particular photovoltaic cell;

normalized luminescence spectrum in the limit of low concentration (no self-absorption);

fill factor or curve factor for a PVC;

flux gain of an LSC: the ratio between the number of photons with energies higher than the bandgap energy transmitted into the PVC with a concentrator as opposed to without a concentrator;

geometric gain of an LSC: the ratio of $A_{f}$ to $A_{e}$;

thickness of an antireflection coating;

Planck's constant divided by $2 \pi$; integrated solar flux per unit area in the AM1 spectrum;

total flux per unit area arriving at the LSC-PVC interface as a function of angle of incidence to the PVC;

flux per wavenumber interval per unit area arriving at the LSC-PVC interface as a function of angle of incidence to the PVC;

saturation current of a PVC;

short circuit current of a PVC;

rate of excitation of the dye ensemble per unit area of the LSC;

width of a PSC;

path length in an LSC traversed by the incident sunlight;

index of refraction of the matrix material;

total population of a two state system;

solar flux per wave number and per unit area for an AM1 spectrum;

ground state population in a two state system;

excited state population in a two state system;

average number of emission undergone by photons in an LSC which arrive at the LSC-PVC interface;

fraction of luminescence that escapes out of the critical cones;

power input to the solar cells per wave number and per unit area;

the fraction of the absorbed solar photons that are luminesced, trapped by total internal reflection, and transported to the LSC-PVC interface; 


\section{$Q^{(n)}$}

$r$

$\bar{r}$

$R\left(\theta_{i}\right), R\left(\theta_{i}, \bar{\nu}\right) \quad$ Fresnel reflection coefficients for an angle of incidence $\theta_{i}$ and wavenumber $\bar{\nu}$ without and with an antireflection coating;

$R_{\mathrm{pvc}}$

$S$

$T\left(\theta_{i}\right), T\left(\theta_{i}, \bar{\nu}\right) \quad$ Fresnel transmission coefficients for an angle of incidence $\theta_{i}$ and wave number $\bar{\nu}$ without and with an antireflection coating;

$T_{\text {pve }} \quad$ average transmission coefficient at the LSC-PVC interface;

$T_{0_{\mathrm{pvc}}}$

$t$

$U\left(\theta_{i}, \bar{\nu}\right)$

V.F.

$V_{\text {oc }}$

W

$W_{12}, W_{21}$

$\alpha(\bar{\nu})$

$\alpha_{m}(\bar{\nu})$

$\alpha_{t}(\bar{\nu})$

$\delta$

$\eta$

$\eta_{\mathrm{lsc}}$

$\eta_{\mathrm{pvc}}$

the ratio of the number of photons that arrive at the LSC-PVC interface difrom their $n$th luminescence to sorbed by the LSC;

average probability that a photon emitted outside of the critical cones self-absorbed;

average probability that a photon emitted inside of the critical cones will average reflection coefficient at the LSC-PVC interface;

solar photon flux absorbed per unit transmission coefficient for sunlight directly incident upon a PVC;

temperature

arbitrary normalized angular and wavenumber distribution of sunlight; voltage factor or perfection factor of a PVC;

open circuit voltage of a PVC;

total flux incident on the LSC-PVC interface;

total probability of excitation and deexcitation per second, respectively, in a two state system;

dye absorption coefficient in inverse centimeters;

matrix material absorption coefficient;

total absorption coefficient for the LSC material;

fraction of the luminescent photons lost to matrix absorption, scattering centers, and incomplete internal reflections due to surface roughness during transport of the photons to the PVC;

molar extinction coefficient for the dye in liters $/ \mathrm{mole} / \mathrm{cm}$;

quantum efficiency of luminescence from an isolated dye molecule or inorganic ion;

total system efficiency of an LSC, or the ratio of the electrical energy output to the solar energy input into the system;

electrical efficiency of a solar cell under direct insolation;
$\theta_{c}$
$\theta_{i}$
$\Lambda(\bar{x}, \bar{y}, \bar{\nu})$
$\xi$
critical angle, $\sin ^{-1}(1 / n)$;
angle of incidence of sunlight on the LSC surface; unit volume that an emission at a po- sition $\bar{y}$ and wave number $\bar{\nu}$ will be ab- sorbed at a position $\bar{x}$; filter function: the probability per
$n$th excitation distribution function: the spatial and wave number distri- bution of the absorbed excitations that will yield the $n$th generation of lumi- nescence;
the fraction of the total solar flux in the AM1 spectrum that is higher in energy than the absorption edge for a partic- ular solar cell.

\section{References}

1. B. A. Swartz, T. Cole, and A. H. Zewail, Opt. Lett. 1, 73 (1977).

2. K. W. Boer, Solar Energy 19, 525 (1977).

3. B. Goldberg and W. H. Klein, Solar Energy 19, 3 (1977).

4. M. Born and E. Wolf, Principles of Optics (Pergamon, New York, 1965), p. 63.

5. A. Goetzberger and W. Greubel, Appl. Phys. 14, 123 (1977).

6. H. J. Hovel, Solar Cells, Vol. 11, Semiconductors and Semimetals (Academic, 1975), New York.

7. K. H. Drexhage, "Structure and Properties of Laser Dyes," in Dye Lasers, Vol. 1, Topics in Applied Physics (Springer, New York, 1977), p. 168.

8. A. Budo and I. Ketskemety, J. Chem. Phys. 25, 595 (1956).

9. J. B. Birks, Photophysics of Aromatic Molecules (Wiley, New York, 1970), p. 42.

10. W. H. Weber and J. Lambe, Appl. Opt. 15, 2299 (1976).

11. L. G. Rainhart and W. P. Schimmel, Jr., Solar Energy 17, 259 (1975).

12. M. Wolf, Proc. IRE 48, 1246 (1960).

13. W. Shockley, Electrons and Holes in Semiconductors (Van Nostrand, New York, 1954), p. 309.

14. C. J. Sah, R. N. Noyce, and W. Shockley, Proc. IRE 45, 1228 (1957).

15. A. G. Chynoweth and K. G. McKay, Phys. Rev. 106, 418 (1957).

16. M. Uman, Introduction to the Physics of Electronics (Prentice-Hall, Englewood Cliffs, N.J., 1957), p. 418.

17. J. Weber, Phys. Lett. A, 57, 465 (1976).

18. D. Beer and J. Weber, Opt. Commun. 5, 307 (1972).

19. J. Weber, Opt. Commun. 7, 420 (1973).

20. Acrilex Inc., 8 Hope Street, Jersey City, N.J., 07307.

21. Q-Panel Company, 15610 Industrial Parkway, Cleveland, Ohio 44135.

22. G. Grossman, J. Coating Technol. 49, 45 (1977).

23. A. Goetzberger, Appl. Phys. 16, 399 (1978).

24. Solar Cell Array Design Handbook, Vol. 1, Jet Propulsion Laboratory Publication JPL SP 43-38, p. 3.1.2 (1976).

25. R. M. Lerner, private communication.

26. J. A. Levitt and W. H. Weber, Appl. Opt. 16, 2684 (1977).

27. G. Bauer and W. Greubel, Appl. Phys. Lett. 31, 4 (1977).

28. W. A. Shurcliff, J. Opt. Soc. Am. 41, 209, (1951).

29. W. A. Shurcliff and R. C. Jones, J. Opt. Soc. Am. 39, 912 (1949).

30. R. L. Garwin, Rev. Sci. Instrum. 31, 1010 (1960).

31. G. Keil, J. Appl. Phys. 40, 3544 (1969).

32. T. C. Weekes, private communication. 